\title{
Canonical Discontinuous Planar Piecewise Linear Systems*
}

\author{
Emilio Freire ${ }^{\dagger}$, Enrique Ponce ${ }^{\dagger}$, and Francisco Torres ${ }^{\dagger}$
}

\begin{abstract}
The family of Filippov systems constituted by planar discontinuous piecewise linear systems with two half-plane linearity zones is considered. Under generic conditions that amount to the boundedness of the sliding set, some changes of variables and parameters are used to obtain a Liénard-like canonical form with seven parameters. This canonical form is topologically equivalent to the original system if one restricts one's attention to orbits with no points in the sliding set. Under the assumption of focus-focus dynamics, a reduced canonical form with only five parameters is obtained. For the case without equilibria in both open half-planes we describe the qualitatively different phase portraits that can occur in the parameter space and the bifurcations connecting them. In particular, we show the possible existence of two limit cycles surrounding the sliding set. Such limit cycles bifurcate at certain parameter curves, organized around different codimension-two Hopf bifurcation points. The proposed canonical form will be a useful tool in the systematic study of planar discontinuous piecewise linear systems, in which this paper is a first step.
\end{abstract}

Key words. Filippov systems, normal form, limit cycle, piecewise linear differential systems

AMS subject classifications. $34 \mathrm{C} 05,34 \mathrm{C} 07,37 \mathrm{G} 15$

DOI. $10.1137 / 11083928 \mathrm{X}$

1. Introduction. Piecewise linear (PWL) systems constitute a class of differential systems which are widely used to model many real processes and different devices; see, for instance, $[1,5,17]$. The case of continuous PWL systems with two linearity regions separated by a straight line is the simplest possible configuration in piecewise smooth systems. Such a family of systems was completely studied in a previous paper [8], where in particular the existence of at most one limit cycle was established.

Enforced by modern nonlinear engineering problems and mathematical biology (see [5]), some recent works in the literature on planar PWL systems deal with vector fields where continuity at the common boundary is not assumed. After the pioneering work of Filippov [7], one must cite in this context the papers of Coll, Gasull, and Prohens [3], Giannakopoulos and Pliete [9], Huan and Yang [11], Kuznetsov, Rinaldi, and Gragnani [13], Llibre, Ponce, and Torres [15], Shui, Zhang, and Li [18], and the recent thorough work of Guardia, Seara, and Teixeira [6], among others.

As mentioned in the recent work of Huan and Yang [11], the study of discontinuous PWL systems is a difficult task because of the lack of a canonical form that can cope with a sufficiently broad class of systems, in contrast to what can be done for the continuous case; see [2]. In fact, only very particular cases are thoroughly analyzed in the available literature.

\footnotetext{
${ }^{*}$ Received by the editors July 1, 2011; accepted for publication (in revised form) by J. Sieber November 15, 2011; published electronically January 31, 2012. This work was partially supported by MICINN/FEDER grant MTM200907849 and by grant EXC/P08-FQM-03770 of Junta de Andalucía.

http://www.siam.org/journals/siads/11-1/83928.html

†Dpto. Matemática Aplicada II, Universidad de Sevilla, Escuela Superior Ingeniería, Camino de los Descubrimientos, Sevilla 41092, Spain (efrem@us.es, eponcem@us.es, ftorres@us.es).
} 
For instance, in [9] the two linear systems share the linear part; in [10] both linear parts are simultaneously in real Jordan canonical form of focus type, which is a nongeneric situation; in [11] the two involved linear systems share the same equilibrium point; and in [15] only Liénard systems with neither real equilibria nor sliding set were considered.

One of the main contributions of this paper is the definition of a Liénard-like canonical form for general discontinuous planar PWL systems with two linear regions separated by a straight line. To fix ideas, let us assume without loss of generality that the linearity regions in the phase plane are the left and right half-planes,

$$
S^{-}=\{(x, y): x<0\}, \quad S^{+}=\{(x, y): x>0\},
$$

so that $x=0$ is the separation line and the systems to be studied become

$$
\dot{\mathbf{x}}=\mathbf{F}(\mathbf{x})= \begin{cases}\mathbf{F}^{+}(\mathbf{x})=\left(F_{1}^{+}(\mathbf{x}), F_{2}^{+}(\mathbf{x})\right)^{T}=A^{+} \mathbf{x}+\mathbf{b}^{+} & \text {if } \mathbf{x} \in S^{+}, \\ \mathbf{F}^{-}(\mathbf{x})=\left(F_{1}^{-}(\mathbf{x}), F_{2}^{-}(\mathbf{x})\right)^{T}=A^{-} \mathbf{x}+\mathbf{b}^{-} & \text {if } \mathbf{x} \in S^{-},\end{cases}
$$

where $\mathbf{x}=(x, y)^{T} \in \mathbb{R}^{2}, A^{+}=\left(a_{i j}^{+}\right)$and $A^{-}=\left(a_{i j}^{-}\right)$are $2 \times 2$ constant matrices, and $\mathbf{b}^{+}=\left(b_{1}^{+}, b_{2}^{+}\right)^{T}$ and $\mathbf{b}^{-}=\left(b_{1}^{-}, b_{2}^{-}\right)^{T}$ are constant vectors of $\mathbb{R}^{2}$. These systems have twelve parameters, and they will be denoted DPWL (discontinuous PWL). When the condition $\mathbf{F}^{+}(0, y)=\mathbf{F}^{-}(0, y)$ is fulfilled for every value of $y$, then systems (1.1) turn out to be continuous, and they have been thoroughly studied in [8], where it was shown that only four parameters are needed for the analysis. Therefore, in this paper we will assume that systems (1.1) do not satisfy the continuity assumption; that is, the condition

$$
A^{+}\left(\begin{array}{c}
0 \\
y
\end{array}\right)+\mathbf{b}^{+} \neq A^{-}\left(\begin{array}{l}
0 \\
y
\end{array}\right)+\mathbf{b}^{-}
$$

generically holds at the $y$-axis.

For systems (1.1) fulfilling the generic condition (1.2), we will propose a canonical form which is able to cover all the cases where self-sustained oscillations are possible. Thus, we will also impose the additional condition $\left|a_{12}^{+}\right|+\left|a_{12}^{-}\right| \neq 0$; otherwise the $x$-component of $\mathbf{F}$ depends only on variable $x$, and so oscillations are precluded because no orbit can cross any line $x=$ const. twice in opposite directions. While the original family needs twelve parameters, our canonical form will require only seven parameters. Furthermore, the existence and characterization of the sliding set is determined by only one of these parameters. This canonical form allows us to obtain a necessary condition for existence of invariant closed curves and also permits us to develop a systematic analysis of the possible dynamical behaviors to be found in planar DPWL systems.

Next, as a first step in such a systematic approach, we partially study in this paper the interesting case of discontinuous coupling of two linear dynamics of focus type. In this case, despite the possible reduction in the number of parameters to five, the complete analysis is rather involved, as will be shown. Consequently, we restrict our attention to the case when there are no real equilibrium points in the interior of each half-plane. To keep the paper within a reasonable length, the remaining cases of the canonical form will be considered elsewhere.

Under these assumptions of focus dynamics without inner equilibria in the linearity regions, the possible limit cycles share no points with the sliding set, and so they can be properly

Copyright (c) by SIAM. Unauthorized reproduction of this article is prohibited. 
called crossing limit cycles. We characterize some bifurcations leading to the birth of crossing periodic orbits and localize certain parameter regions where the number of crossing limit cycles is exactly two. Several bifurcation sets are obtained as by-products of the different theoretical results.

In particular, apart from standard codimension-one bifurcations (see [13]), we find different codimension-two bifurcation points, namely a degenerate Hopf bifurcation at infinity and a degenerate pseudo-Hopf bifurcation at the origin. Hopf bifurcation at infinity for PWL systems has been studied in [14] but only for specific continuous cases; anyway, this bifurcation deserves a deeper analysis not to be included here.

Pseudo-Hopf bifurcations are well known (see, for instance, $[6,13])$, but the unfolding of this degenerate case also needs more attention. The results that we obtain, when compared with those included in [6], make evident the important role of DPWL systems as normal forms in the study of local bifurcations of piecewise smooth systems; see the final comments in section 4 .

There seemingly appears a third codimension-two bifurcation point involving the two previous bifurcations in nondegenerate situations; see section 4 . We conjecture that the two degenerate bifurcation points are linked by a curve which bounds the parameter region where two crossing limit cycles coexist, two being the maximum number of crossing periodic orbits. We remark that this maximum number is at least three when equilibrium points are allowed to be in the interior of linearity regions; see [11].

The rest of the paper is outlined as follows. In section 2 we give a short review of Filippov systems with emphasis on our planar systems. In section 3 the promised canonical form and a necessary condition for the existence of periodic orbits are obtained. The main results of this paper and some bifurcation sets are shown in section 4 , where a specific canonical form for the focus-focus case with only five parameters is also given. In section 5 some illustrative examples are reduced to the proposed canonical form, showing the usefulness of our theoretical approach. Several conclusions are outlined in section 6 , whereas the proofs of our main results are relegated to section 7 .

2. DPWL systems as Filippov systems. Although both functions $\mathbf{F}^{+}$and $\mathbf{F}^{-}$are defined in every point of $\mathbb{R}^{2}$, the vector field (1.1) is not explicitly defined when $x=0$, which is the discontinuity locus, and so the definition of a solution for discontinuous system (1.1) needs to be clarified. Following the approach in [13], we will not define solutions $(x(t), y(t))$ with initial values at the $y$-axis, but by considering solutions with $x(0) \neq 0$, we will give a precise sense to the fact that orbits can pass through the discontinuity set.

Clearly, an orbit is well defined while it evolves without touching the $y$-axis. However, we must adopt some criterion to define the orbits arriving at the discontinuity line $\Sigma=\{(x, y)$ : $x=0\}$. As usual, we will adopt the Filippov convex method (see [13]), so that solutions can be uniquely defined in forward time, although they can be nonsmooth.

The restriction of the system to each one of the zones $x \neq 0$ is a linear system, so that the solutions living in only one zone can be explicitly computed. When a solution arrives at the discontinuity line at the point $(0, y)$ different things can occur.

If $F_{1}^{+}(0, y) F_{1}^{-}(0, y)>0$, then both vector fields are transversal to the discontinuity line, and their normal components have the same sign. In this case, we will assume that orbits

Copyright (c) by SIAM. Unauthorized reproduction of this article is prohibited. 
are concatenated in the natural way. For instance, if an orbit arrives at the discontinuity line coming from the zone $x<0$, that is $F_{1}^{-}(0, y)>0$, then assuming that also $F_{1}^{+}(0, y)>0$, the orbit crosses the line $x=0$ towards the other linearity zone with a discontinuity in the tangent vector. We say that this point is a crossing point, so that the crossing set $\Sigma^{c}$ is defined as follows:

$$
\Sigma^{c}=\left\{(0, y): F_{1}^{+}(0, y) F_{1}^{-}(0, y)>0\right\} .
$$

If $F_{1}^{+}(0, y) F_{1}^{-}(0, y) \leqslant 0$, then either the normal components of vector fields to the discontinuity line have opposite sign or at least one of them vanishes. We speak of $(0, y)$ as a sliding point, and the set

$$
\Sigma^{s}=\left\{(0, y): F_{1}^{+}(0, y) F_{1}^{-}(0, y) \leqslant 0\right\}
$$

is named the sliding set. In this case, we assume that the orbit slides along the sliding set according to the convex method of Filippov; that is, the solutions of our system satisfy the equation

$$
\dot{\mathbf{x}}=\lambda \mathbf{F}^{-}(\mathbf{x})+(1-\lambda) \mathbf{F}^{+}(\mathbf{x}), \quad \mathbf{x} \in \Sigma^{s},
$$

where $\lambda$ is selected so that the above vector field is tangent to the sliding set, i.e.,

$$
\lambda F_{1}^{-}(\mathbf{x})+(1-\lambda) F_{1}^{+}(\mathbf{x})=0, \quad \mathbf{x} \in \Sigma^{s} .
$$

Then, for $\mathbf{x} \in \Sigma^{s}$ and $\left|F_{1}^{+}(\mathbf{x})\right|+\left|F_{1}^{-}(\mathbf{x})\right| \neq 0$, we get

$$
\lambda(y)=\frac{F_{1}^{+}(\mathbf{x})}{F_{1}^{+}(\mathbf{x})-F_{1}^{-}(\mathbf{x})},
$$

and the so-called sliding solutions are given by

$$
\dot{x}=0, \quad \dot{y}=g(y)=\frac{F_{1}^{+}(\mathbf{x}) F_{2}^{-}(\mathbf{x})-F_{1}^{-}(\mathbf{x}) F_{2}^{+}(\mathbf{x})}{F_{1}^{+}(\mathbf{x})-F_{1}^{-}(\mathbf{x})}, \quad \mathbf{x} \in \Sigma^{s} .
$$

Note that our notation differs from that used in [6], where $\Sigma^{s}$ is reserved for the attractive part of the sliding set, that is, when $F_{1}^{+}(0, y)<0$ and $F_{1}^{-}(0, y)>0$; they also use $\Sigma^{e}$ for the escaping or repulsive part of the sliding set, that is, when $F_{1}^{+}(0, y)>0$ and $F_{1}^{-}(0, y)<0$.

A particular instance of a sliding point occurs when $\left|F_{1}^{+}(\mathbf{p})\right|+\left|F_{1}^{-}(\mathbf{p})\right| \neq 0$ but $F_{1}^{+}(\mathbf{p})$. $F_{1}^{-}(\mathbf{p})=0$. In this case the point $\mathbf{p}$ is said to be a tangency point. Assume that $\mathbf{p}$ is a tangency point with $F_{1}^{+}(\mathbf{p})=0$. If the orbit of the vector field $\mathbf{F}^{+}$passing through $\mathbf{p}$ at time $t=t_{p}$ remains in the region $x>0(x<0)$ for $\left|t-t_{p}\right|$ small, then the point $\mathbf{p}$ will be called a visible (invisible) tangency point. Analogously, we can have visible or invisible tangency points by considering the vector field $\mathbf{F}^{-}$.

It is important to observe that since our vector fields $\mathbf{F}^{ \pm}$are linear, under the tangency assumptions $\dot{x}=0$ and $\dot{y} \neq 0$, a simple computation shows that the tangency is quadratic whenever the corresponding coefficient $a_{12}^{ \pm}$does not vanish.

If $F_{1}^{+}(\mathbf{p})=F_{1}^{-}(\mathbf{p})=0$, then the point $\mathbf{p}$ is a singular sliding point, and three cases arise: (1) both vector fields are tangent to the line $x=0,(2)$ one of them is tangent while the other

Copyright $\odot$ by SIAM. Unauthorized reproduction of this article is prohibited. 
one vanishes, and (3) both vector fields vanish. In the first case $\mathbf{p}$ will be called a double tangency point, while in the two last cases $\mathbf{p}$ will be called a boundary equilibrium point.

For our DPWL systems, the double tangency points are isolated points in the discontinuity line. If the point of double tangency has a visible tangency at one side and an invisible tangency at the other side, the quoted point behaves as a regular point. When the tangency is either visible or invisible at both sides of the double tangency point $(0, y)$ we simply impose that $g(y)=0$; for boundary equilibrium points $(0, y)$ we also set $g(y)=0$.

Points $(0, \bar{y}) \in \Sigma^{s}$ with $g(\bar{y})=0$ act in some sense as equilibria of system (1.1), and they are called pseudoequilibria. A double invisible tangency point with close orbits spiraling around it is called a pseudofocus or fused focus; see [13]. A pseudoequilibrium in the attractive part of the sliding set with $g^{\prime}(y)<0$ is a stable pseudonode, being a pseudosaddle if $g^{\prime}(y)>0$. Similarly, a pseudoequilibrium in the repulsive part with $g^{\prime}(y)>0$ is an unstable pseudonode, being again a pseudosaddle if $g^{\prime}(y)<0$. Note that at pseudoequilibria $(0, \bar{y})$ which are neither boundary equilibrium nor tangency points we have

$$
\frac{F_{2}^{-}(0, \bar{y})}{F_{1}^{-}(0, \bar{y})}=\frac{F_{2}^{+}(0, \bar{y})}{F_{1}^{+}(0, \bar{y})}
$$

and so the two vector fields $\mathbf{F}^{+}$and $\mathbf{F}^{-}$are anticollinear.

3. Canonical form and general results for DPWL systems. Since our goal is to study nonlinear phenomena, we look for possible periodic orbits not totally contained in $S^{-}$or $S^{+}$. These orbits must be of one of following two types, depending on the nature of their points on the $y$-axis. If the periodic orbit has sliding points, then it will be called a sliding periodic orbit. Otherwise we speak of crossing periodic orbits, whose study constitutes the main goal of our analysis.

From (2.1), the crossing set of our systems is constituted by the points $(0, y)$ for which the expression

$$
F_{1}^{+}(0, y) F_{1}^{-}(0, y)=\left(a_{12}^{+} y+b_{1}^{+}\right)\left(a_{12}^{-} y+b_{1}^{-}\right)
$$

is positive. When $a_{12}^{-} a_{12}^{+} \leqslant 0$, it is easy to see that the crossing set, if it exists, is an open interval of the $y$-axis, unbounded for $a_{12}^{-} a_{12}^{+}=0$ and bounded for $a_{12}^{-} a_{12}^{+}<0$. In any case, the $x$-component of both vector fields has constant sign at the crossing set, and so elementary qualitative arguments preclude the existence of crossing periodic orbits. These cases will not be considered in the rest of the paper, and we assume in what follows that $a_{12}^{-} a_{12}^{+}>0$, which means that the sliding set is bounded and either attractive or repulsive.

In order to obtain the promised canonical form, we will make a continuous PWL change of variables such that the resulting transformation will be a homeomorphism, keeping invariant the discontinuity line $\Sigma$ and leading to a new vector field with few parameters. The proposed change will also keep invariant the half-planes $S^{+}$and $S^{-}$and, being linear within each open half-plane, the existence in $S^{+} \cup S^{-}$of a conjugacy between both vector fields can be guaranteed. Later on (see Remark 3.2), we will discuss the implications of this change of variables for orbits having points in $\Sigma$, and specially for orbits with points in the sliding set $\Sigma^{s}$.

Copyright (c) by SIAM. Unauthorized reproduction of this article is prohibited. 
Looking for a Liénard-like canonical form for DPWL systems, and denoting by $T^{-}$and $T^{+}$and by $D^{-}$and $D^{+}$the traces and determinants of matrices $A^{-}$and $A^{+}$, respectively, the following result can be stated.

Proposition 3.1 (Liénard canonical form for DPWL systems). Assume that $a_{12}^{+} a_{12}^{-}>0$ in system (1.1). Then the homeomorphism $\widetilde{\mathbf{x}}=h(\mathbf{x})$ given by

$$
\widetilde{\mathbf{x}}=\left(\begin{array}{cc}
1 & 0 \\
a_{22}^{-} & -a_{12}^{-}
\end{array}\right) \mathbf{x}-\left(\begin{array}{c}
0 \\
b_{1}^{-}
\end{array}\right) \quad \text { if } \mathbf{x} \in S^{-} \cup \Sigma
$$

and

$$
\widetilde{\mathbf{x}}=\frac{1}{a_{12}^{+}}\left(\begin{array}{cc}
a_{12}^{-} & 0 \\
a_{12}^{-} a_{22}^{+} & -a_{12}^{-} a_{12}^{+}
\end{array}\right) \mathbf{x}-\left(\begin{array}{c}
0 \\
b_{1}^{-}
\end{array}\right) \quad \text { if } \mathbf{x} \in S^{+}
$$

after dropping tildes, transforms system (1.1) into the canonical form

$$
\begin{aligned}
& \dot{\mathbf{x}}=\mathbf{G}^{-}(\mathbf{x})=\left(\begin{array}{rr}
T^{-} & -1 \\
D^{-} & 0
\end{array}\right) \mathbf{x}-\left(\begin{array}{c}
0 \\
a^{-}
\end{array}\right) \quad \text { if } \mathbf{x} \in S^{-}, \\
& \dot{\mathbf{x}}=\mathbf{G}^{+}(\mathbf{x})=\left(\begin{array}{rr}
T^{+} & -1 \\
D^{+} & 0
\end{array}\right) \mathbf{x}-\left(\begin{array}{c}
-b \\
a^{+}
\end{array}\right) \quad \text { if } \mathbf{x} \in S^{+},
\end{aligned}
$$

where

$$
a^{-}=a_{12}^{-} b_{2}^{-}-a_{22}^{-} b_{1}^{-}, \quad b=\frac{a_{12}^{-}}{a_{12}^{+}} b_{1}^{+}-b_{1}^{-}, \quad a^{+}=\frac{a_{12}^{-}}{a_{12}^{+}}\left(a_{12}^{+} b_{2}^{+}-a_{22}^{+} b_{1}^{+}\right) .
$$

Besides the invariance of the discontinuity line $\Sigma$, the crossing and sliding sets, tangency points, and boundary equilibria of the original system (1.1) are transformed by the homeomorphism $h$ into sets and points of the same type for system (3.4).

Moreover, there is a topological equivalence between systems (1.1) and (3.4) for all their orbits not having points in common with the sliding set. However, the homeomorphism $h$ preserves the attractive or repulsive character of the sliding set.

Proof. To obtain the vector field (3.4) is a direct computation. The assertion about the homeomorphism between the subsets of $\Sigma$, tangency points, and boundary equilibria follows from the equalities

$$
\mathbf{G}_{1}^{+}(h(0, y))=\left(a_{12}^{-} y+b_{1}^{-}+b\right)=\frac{a_{12}^{-}}{a_{12}^{+}} \mathbf{F}_{1}^{+}(0, y), \quad \mathbf{G}_{1}^{-}(h(0, y))=\left(a_{12}^{-} y+b_{1}^{-}\right)=\mathbf{F}_{1}^{-}(0, y) .
$$

Thus the signs of the $x$-components are preserved, and so the product $\mathbf{G}_{1}^{+}(h(0, y)) \mathbf{G}_{1}^{-}(h(0, y))$ is equal to expression (3.1) up to a positive factor. Obviously, orbits totally contained in one of the two open half-planes are transformed in a homeomorphic way. Moreover, since orbits arriving at the crossing set are continued by the natural concatenation, the topological equivalence is not lost at the crossing set.

Before proceeding further, some important observations are in order.

Remark 3.2. The change of variables used in the above proof is a homeomorphism defined in the whole plane leading to a linear conjugacy for each vector field in $S^{+}$and $S^{-}$, separately.

Copyright (C) by SIAM. Unauthorized reproduction of this article is prohibited. 
As indicated, the topological equivalence can be extended to the crossing set, so that crossing periodic orbits in the initial system are also transformed, in a homeomorphic way, into crossing periodic orbits of the canonical form. In studying crossing limit cycles, this observation is crucial for understanding the importance of the introduced canonical form.

In general, the restriction of this homeomorphism to the discontinuity line $\Sigma$ does not produce a conjugacy between the corresponding sliding vector fields. In fact, the change (3.2)(3.3) is nonsmooth and, as indicated in Remark 2.24 from [6], one cannot guarantee topological equivalence between sliding vector fields. Effectively, it is easy to build examples where the number of pseudoequilibria changes in passing to the proposed canonical form.

This lack of equivalence between the two sliding vector fields is not relevant if one is interested in the dynamics not involving sliding orbits, as is the case for the analysis of crossing periodic orbits to be made in what follows. Therefore, the different definitions of equivalence given in [6] apply whenever we except the sliding set $\Sigma^{s}$. The price to be paid if one is interested in preserving the sliding dynamics is to use a diffeomorphic change; for instance, by extending the change in (3.2) to the whole plane, the left vector field obtained is the same as in (3.4), but the simplest expression for the right vector field should become

$$
\dot{\mathbf{x}}=\left(\begin{array}{cc}
g_{11}^{+} & -1 \\
g_{21}^{+} & g_{22}^{+}
\end{array}\right) \mathbf{x}-\left(\begin{array}{c}
-b \\
\widetilde{a}^{+}
\end{array}\right) \quad \text { if } \mathbf{x} \in S^{+}
$$

after a time rescaling for orbits in $S^{+}$. Even if the parameter $b$ is unmodified, this right vector field is not in Liénard form any longer. Obviously, its analysis becomes more involved; in any case, being interested in the crossing dynamics, we will not consider it further.

When $\Sigma^{s}$ becomes one point, there is no sliding dynamics, and the previous remark is not relevant. In such a case, system (1.1) and (3.4) are trivially $\Sigma$-equivalent according to the definition given in [6], but the following observation is noteworthy.

Remark 3.3. Note that if $b=0$ and $a^{-}=a^{+}$, then system (3.4) turns out to be continuous. Thus Proposition 3.1 assures that certain discontinuous systems (1.1) can be transformed into continuous systems by means of a continuous PWL change of variables. More precisely, if a discontinuous system (1.1) satisfies

$$
a_{12}^{+} a_{12}^{-}>0, \quad b_{1}^{-}=\frac{a_{12}^{-}}{a_{12}^{+}} b_{1}^{+}, \quad b_{2}^{-}-\frac{a_{22}^{-}}{a_{12}^{-}} b_{1}^{-}=b_{2}^{+}-\frac{a_{22}^{+}}{a_{12}^{+}} b_{1}^{+},
$$

then its corresponding canonical form (3.4) is continuous. In particular, we see that homogeneous systems (1.1) with $a_{12}^{+} a_{12}^{-}>0$ and $\mathbf{b}^{+}=\mathbf{b}^{-}=0$ can always be transformed in a continuous system. Thus, for instance, the class of bimodal systems considered in [12] and the analysis done in [20] are not necessary, since they can be completely recast into the continuous cases studied in [8].

Thus, from the above remark, some presumed Filippov systems can be considered as false discontinuous systems. This fact is a surprising byproduct of the above canonical form. We emphasize that the study of the properties of the family of systems (3.4), which represents an important subset of all possible DPWL systems, is relevant on its own. We claim that the family is able to reproduce most of the nonlinear dynamics one can find in DPWL systems. Here, we start its thorough study by considering a useful reduction in the number of different cases to be analyzed.

Copyright (c) by SIAM. Unauthorized reproduction of this article is prohibited. 
Remark 3.4. Canonical form (3.4) is invariant under the change of variables $(x, y, t) \rightarrow$ $(x,-y,-t)$, simultaneous with the change of parameters

$$
\left(D^{+}, D^{-}, T^{+}, T^{-}, a^{+}, a^{-}, b\right) \rightarrow\left(D^{+}, D^{-},-T^{+},-T^{-}, a^{+}, a^{-},-b\right) .
$$

Thus, it is enough to consider $b \geqslant 0$ in the study of system (3.4).

In the following, according to Remark 3.4, we concentrate our attention on the canonical form (3.4) with $b \geqslant 0$. Next, we give some preliminary results related to the existence of crossing periodic orbits.

From (2.2), the sliding set of the canonical form (3.4) is determined by the inequality

$$
F_{1}^{-}(0, y) F_{1}^{+}(0, y)=y(y-b) \leqslant 0 .
$$

Since $b \geqslant 0$, the sliding set is the segment

$$
\Sigma^{s}=\{(x, y): x=0, \quad 0 \leqslant y \leqslant b\},
$$

shrinking to the origin when $b=0$. When $b>0$, the convex method of Filippov (see $(2.3)$ ) leads to the system

$$
\dot{x}=0, \quad \dot{y}=\frac{a^{-}-a^{+}}{b} y-a^{-}, \quad 0 \leqslant y \leqslant b .
$$

As the normal component of both vector fields points outwards from the sliding set when $b>0$, we say that the sliding set is unstable in the normal direction. The crossing set is the complement in $\Sigma$ of the sliding set, i.e.,

$$
\Sigma^{c}=\{(x, y): x=0, y<0\} \cup\{(x, y): x=0, b<y\} .
$$

Regarding the tangency points and pseudoequilibrium points of system (3.4), we give the next proposition, whose proof is elementary and will not be shown.

Proposition 3.5. For system (3.4) the following statements hold:

(a) If $a^{-} \neq 0$, then the origin is a left tangency point, visible for $a^{-}<0$ and invisible for $a^{-}>0$. If $a^{-}=0$, then the origin is a boundary equilibrium point.

(b) If $a^{+} \neq 0$, then the point $(0, b)$ is a right tangency point, visible for $a^{+}>0$ and invisible for $a^{+}<0$. If $a^{+}=0$, then the point $(0, b)$ is a boundary equilibrium point.

(c) If $b=0$ and $a^{+} a^{-} \neq 0$, then the origin is a double tangency point. It behaves as a regular point for $a^{+} a^{-}>0$, being a pseudoequilibrium point for $a^{+} a^{-}<0$; in fact, the origin is a pseudofocus for $a^{+}<0<a^{-}$and a pseudosaddle for $a^{-}<0<a^{+}$.

(d) For $b>0$, regarding possible pseudoequilibrium points in the interior of the sliding set, the following subcases arise:

(d1) If $a^{-}=a^{+}=0$, then every point of the interior of the sliding set is a pseudoequilibrium point.

(d2) If $a^{-} a^{+}<0$, then the point $\left(0, \frac{a^{-} b}{a^{-}-a^{+}}\right)$is the only pseudoequilibrium point, being a pseudosaddle for $a^{-}<0$ and an unstable pseudonode for $a^{-}>0$.

(d3) If $a^{+} a^{-} \geqslant 0$ with $a^{-}+a^{+} \neq 0$, then there are no pseudoequilibrium points in the interior of the sliding set.

Copyright (c) by SIAM. Unauthorized reproduction of this article is prohibited. 


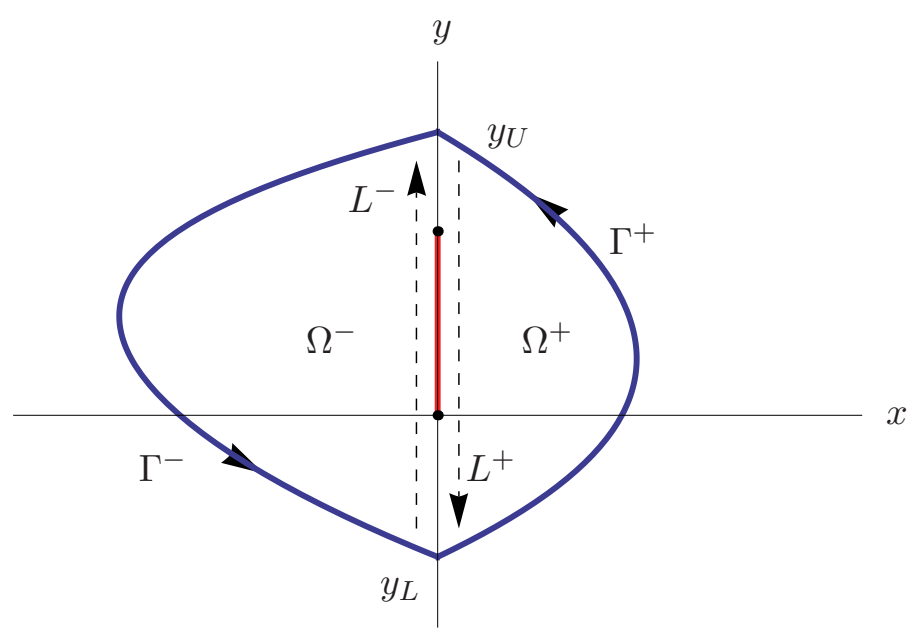

Figure 1. A crossing periodic orbit surrounding the sliding set.

Note that the above statements for tangency points can be translated back to the original system corresponding to the canonical form, but the same is not true for pseudoequilibria; see Remark 3.2. Regarding the possible equilibria of system (3.4) belonging to the open sets $S^{-}$ and $S^{+}$, the following standard proposition is also given without proof.

Proposition 3.6. The following statements hold:

(a) System (3.4) has one equilibrium point in the left zone when $a^{-} D^{-}<0$, and no equilibria in that zone when $a^{-} D^{-}>0$. Analogously, it has one equilibrium point in the right zone when $a^{+} D^{+}>0$, and no equilibria in that zone when $a^{+} D^{+}<0$.

(b) If $D^{-}=0$, then the system has no equilibria in the left zone when $a^{-} \neq 0$, having a continuum of equilibria making up the half-straight line $y=T^{-} x, x<0$, when $a^{-}=0$. Analogously, if $D^{+}=0$, then the system has no equilibria in the right zone when $a^{+} \neq 0$, having a continuum of equilibria making up the half-straight line $y=T^{+} x-b, x>0$, when $a^{+}=0$.

Since for $y>b$ we have $F_{1}^{+}(0, y)<0$ and $F_{1}^{-}(0, y)<0$ and for $y<0$ we have $F_{1}^{+}(0, y)>0$ and $F_{1}^{-}(0, y)>0$, we conclude that a crossing periodic orbit $\Gamma$ has exactly two points at the $y$-axis, namely, the two points $\left(0, y_{L}\right)$ and $\left(0, y_{U}\right)$ with $y_{L}<0<b<y_{U}=y_{L}+h$ with $h>0$; see Figure 1.

Here, a necessary condition for the existence of crossing periodic orbits in system (1.1) will be given. We define, by removing the two crossing points, the left open $\operatorname{arc} \Gamma^{-}=\Gamma \cap S^{-}$, the right open $\operatorname{arc} \Gamma^{+}=\Gamma \cap S^{+}$, and the oriented segments

$$
L^{-}=\left\{(x, y): x=0, y=(1-\mu) y_{L}+\mu y_{U}, 0 \leqslant \mu \leqslant 1\right\}
$$

and

$$
L^{+}=\left\{(x, y): x=0, y=\mu y_{L}+(1-\mu) y_{U}, 0 \leqslant \mu \leqslant 1\right\} .
$$

Since $\Gamma^{-} \cup L^{-}$is a closed Jordan curve, its interior $\Omega^{-}=\operatorname{int}\left\{\Gamma^{-} \cup L^{-}\right\}$and the value $\sigma^{-}=$ area $\left(\Omega^{-}\right)$are well defined. Analogously, it can be considered that $\Omega^{+}=\operatorname{int}\left\{\Gamma^{+} \cup L^{+}\right\}$and 
$\sigma^{+}=\operatorname{area}\left(\Omega^{+}\right)$. Next, the following proposition, whose proof is given in section 7 , is deduced from Green's formula.

Proposition 3.7. If system (3.4) has a crossing periodic orbit $\Gamma$ that crosses the line $x=0$ through the points $\left(0, y_{L}\right)$ and $\left(0, y_{L}+h\right)$, where $h>0$, then

$$
T^{-} \sigma^{-}+T^{+} \sigma^{+}+b h=0 .
$$

The above result, as a necessary condition for the existence of periodic orbits, is also useful to exclude them in certain cases; see the next remark.

Remark 3.8. We remark that in the two cases (i) $T^{+}>0, T^{-}>0, b>0$, and (ii) $T^{+} T^{-} \geqslant 0, T^{+}+T^{-} \neq 0, b=0$, the equality (3.7) of Proposition 3.7 cannot be fulfilled, and so system (3.4) cannot have crossing periodic orbits.

4. The focus-focus case without equilibria in the interior of the half-planes. In the remainder, we pay attention only to one of the most important cases in practice, in which both dynamics are of focus type; that is, $T^{2}-4 D<0$ in both zones. In this case, we can give a simpler canonical form depending on only five parameters.

Proposition 4.1 (Liénard reduced form in the focus-focus case). Assume $T^{ \pm}=2 \alpha^{ \pm}, D^{ \pm}=$ $\left(\alpha^{ \pm}\right)^{2}+\left(\omega^{ \pm}\right)^{2}$ with $\omega^{ \pm}>0$ in the canonical form (3.4), so that the corresponding eigenvalues are $\lambda^{ \pm}=\alpha^{ \pm} \pm i \omega^{ \pm}$, and introduce the parameters

$$
\gamma_{R}=\frac{\alpha^{+}}{\omega^{+}}, \quad \gamma_{L}=\frac{\alpha^{-}}{\omega^{-}}, \quad a_{R}=\frac{a^{+}}{\omega^{+}}, \quad a_{L}=\frac{a^{-}}{\omega^{-}} .
$$

Then the change of variables (different for each half-plane)

$$
(x, y, t) \rightarrow\left(\frac{x}{\omega(x)}, y, \frac{t}{\omega(x)}\right), \quad \text { where } \omega(x)=\left\{\begin{array}{l}
\omega^{-} \text {if } x<0 \\
\omega^{+} \text {if } x>0
\end{array}\right.
$$

transforms the canonical form (3.4) into the form

$$
\begin{gathered}
\dot{\mathbf{x}}=\left(\begin{array}{cr}
2 \gamma_{L} & -1 \\
1+\gamma_{L}^{2} & 0
\end{array}\right) \mathbf{x}-\left(\begin{array}{c}
0 \\
a_{L}
\end{array}\right) \quad \text { if } \mathbf{x} \in S^{-}, \\
\dot{\mathbf{x}}=\left(\begin{array}{cr}
2 \gamma_{R} & -1 \\
1+\gamma_{R}^{2} & 0
\end{array}\right) \mathbf{x}-\left(\begin{array}{c}
-b \\
a_{R}
\end{array}\right) \quad \text { if } \mathrm{x} \in S^{+} .
\end{gathered}
$$

Using again Remark 3.2, the study of crossing periodic orbits of system (3.4) can be done by means of the analysis of system (4.1). However, the rich possible dynamics of system (4.1) forces us to split its study into different steps. As the first step, here we will consider only the case where there are no real equilibria in the open zones $S^{+}$and $S^{-}$; that is, we assume in the following that $a_{R} \leqslant 0 \leqslant a_{L}$. From Proposition 3.5, the endpoints of the sliding set are invisible tangency points or boundary equilibria. The cases where there can be equilibria in the interior of the half-planes will be studied in a forthcoming paper.

Our first main results are related to the case $b=0$. This situation was already considered in [15] but only when $a_{R}<0<a_{L}$ and $\gamma_{R} \gamma_{L} \neq 0$. Here, we present new results including all the possible situations. Clearly, the origin is then the single sliding point and is always a

Copyright (c) by SIAM. Unauthorized reproduction of this article is prohibited. 
topological focus, whose Lyapunov constants could be obtained through the analysis done in [3]; here we follow a different approach to capture not only local but also global information.

Proposition 4.2 (stability of the origin in systems without sliding set). Assuming $b=0$ and $a_{R} \leqslant 0 \leqslant a_{L}$ in system (4.1), the following statements hold:

(a) The origin is asymptotically stable for $a_{L} \gamma_{R}<a_{R} \gamma_{L}$ and unstable for $a_{L} \gamma_{R}>a_{R} \gamma_{L}$.

(b) If $a_{L} \gamma_{R}=a_{R} \gamma_{L}$, then the origin is unstable for $\gamma_{R}+\gamma_{L}>0$, asymptotically stable for $\gamma_{R}+\gamma_{L}<0$, and a global center for $\gamma_{R}+\gamma_{L}=0$.

It is worth noting that, under the assumptions of Proposition 4.2(b), if $\gamma_{R}=-\gamma_{L} \neq 0$, then $a_{R}=-a_{L} \neq 0$, and the system is reversible. The above conditions allow us to identify the DPWL systems which have reversible nonlinear global centers.

Theorem 4.3 (systems without sliding set). Assuming in system (4.1) the conditions $b=0$ and $a_{R} \leqslant 0 \leqslant a_{L}$, the following statements hold:

(a) If $\gamma_{R}+\gamma_{L}=0$, then there is a global nonlinear center around the origin for $a_{L} \gamma_{R}=$ $a_{R} \gamma_{L}$, and no crossing periodic orbits when $a_{L} \gamma_{R} \neq a_{R} \gamma_{L}$.

(b) If $\gamma_{R}+\gamma_{L} \neq 0$ and $\gamma_{R} \gamma_{L} \geqslant 0$, then there are no crossing periodic orbits.

(c) If $\gamma_{R}+\gamma_{L} \neq 0$ and $\gamma_{R} \gamma_{L}<0$, then for $\left(\gamma_{R}+\gamma_{L}\right)\left(a_{L} \gamma_{R}-a_{R} \gamma_{L}\right)<0$ there is only one crossing periodic orbit, which is stable for $\gamma_{R}+\gamma_{L}<0$ and unstable for $\gamma_{R}+\gamma_{L}>0$. When $\left(\gamma_{R}+\gamma_{L}\right)\left(a_{L} \gamma_{R}-a_{R} \gamma_{L}\right) \geqslant 0$ there are no crossing periodic orbits.

Corollary 4.4 (global asymptotic stability of the origin). Under the conditions $b=0$ and $a_{R}$ $\leqslant 0 \leqslant a_{L}$, the origin in system (4.1) is globally asymptotically stable in the two following cases: (i) when $a_{L} \gamma_{R}<a_{R} \gamma_{L}$ and $\gamma_{R}+\gamma_{L} \leqslant 0$, and (ii) when $a_{L} \gamma_{R}=a_{R} \gamma_{L}$ and $\gamma_{R}+\gamma_{L}<0$.

For $b>0$ in system (4.1), we have that the normal components of both vector fields at the set $\Sigma^{s}$ defined in (3.5) point outwards, so the sliding set is unstable in the normal direction. Then the orbits that leave the sliding set never return to it, and we cannot have stable sliding periodic orbits. If additionally $a_{R}<0<a_{L}$, then from Proposition 3.5(d2) an unstable pseudonode appears in the sliding set. Then, by reversing time, the orbits entering into the sliding set go towards the pseudonode, which now becomes stable. Thus our system has no unstable sliding periodic orbits either. In the limiting case $a_{R} a_{L}=0$, we have at least one boundary equilibrium point, and a similar argument precludes the existence of sliding periodic orbits. Therefore, we focus our attention on the existence and number of crossing periodic orbits for $b \neq 0$.

Theorem 4.5 (systems with escaping sliding set, $b>0$ ). Assuming the conditions $a_{R} \leqslant 0 \leqslant$ $a_{L}, b>0$ in system (4.1), the following statements hold:

(a) If $\gamma_{R} \gamma_{L} \geqslant 0$, then there are no crossing periodic orbits for $\gamma_{R}+\gamma_{L} \geqslant 0$, while for $\gamma_{R}+\gamma_{L}<0$ there is only one crossing periodic orbit, which is stable.

(b) If $\gamma_{R} \gamma_{L}<0$, then the following subcases arise:

(b1) If $\gamma_{R}+\gamma_{L} \geqslant 0$ and $a_{L} \gamma_{R} \geqslant a_{R} \gamma_{L}$, then there are no crossing periodic orbits.

(b2) If $\gamma_{R}+\gamma_{L}=0, a_{L} \gamma_{R}<a_{R} \gamma_{L}$, and we define the value

$$
b_{\infty}=2\left(a_{L}+a_{R}\right) \frac{\gamma_{L}}{1+\gamma_{L}^{2}}=-2\left(a_{L}+a_{R}\right) \frac{\gamma_{R}}{1+\gamma_{R}^{2}},
$$

then $b_{\infty}>0$, and there is only one crossing periodic which is stable for $0<b<b_{\infty}$ and no periodic orbits for $b \geqslant b_{\infty}$.

(b3) If $\gamma_{R}+\gamma_{L}>0$ and $a_{L} \gamma_{R}<a_{R} \gamma_{L}$, then there are two hyperbolic crossing periodic

Copyright $\odot$ by SIAM. Unauthorized reproduction of this article is prohibited. 
orbits for $b$ sufficiently small, while there are no crossing periodic orbits for $b$ sufficiently big.

If additionally $a_{R} a_{L}=0$ with $a_{R}+a_{L} \neq 0$, there exists a value $b_{S N}$ such that (4.1) has exactly two hyperbolic crossing periodic orbits for $0<b<b_{S N}$, only one crossing periodic orbit which is semistable for $b=b_{S N}$, and no crossing periodic orbits for $b>b_{S N}$.

(b4) If $\gamma_{R}+\gamma_{L}<0$, then there is always a stable crossing periodic orbit. If in addition $a_{R} a_{L}=0$, then the above crossing periodic orbit is unique.

The following theorem is a direct consequence of the previous one, after using Remark 3.4. Theorem 4.6 (systems with attractive sliding set, $b<0$ ). Assuming the conditions $a_{R} \leqslant 0 \leqslant$ $a_{L}$ and $b<0$ in system (4.1), the following statements hold:

(a) If $\gamma_{R} \gamma_{L} \geqslant 0$, then for $\gamma_{R}+\gamma_{L} \leqslant 0$ there are no crossing periodic orbits, while for $\gamma_{R}+\gamma_{L}>0$ there is only one crossing periodic orbit, which is unstable.

(b) If $\gamma_{R} \gamma_{L}<0$, then the following subcases arise:

(b1) If $\gamma_{R}+\gamma_{L} \leqslant 0$ and $a_{L} \gamma_{R} \leqslant a_{R} \gamma_{L}$, then there are no crossing periodic orbits.

(b2) If $\gamma_{R}+\gamma_{L}=0, a_{L} \gamma_{R}>a_{R} \gamma_{L}$, and we define the value

$$
b_{\infty}=2\left(a_{L}+a_{R}\right) \frac{\gamma_{L}}{1+\gamma_{L}^{2}}=-2\left(a_{L}+a_{R}\right) \frac{\gamma_{R}}{1+\gamma_{R}^{2}},
$$

then $b_{\infty}<0$ and there is only one crossing periodic, which is unstable for $b_{\infty}<$ $b<0$, and no periodic orbits for $b \leqslant b_{\infty}$.

(b3) If $\gamma_{R}+\gamma_{L}<0$ and $a_{L} \gamma_{R}>a_{R} \gamma_{L}$, then there are two hyperbolic crossing periodic orbits for $|b|$ sufficiently small, while there are no crossing periodic orbits for $|b|$ sufficiently big.

If additionally $a_{R} a_{L}=0$ with $a_{R}+a_{L} \neq 0$, there exists a value $b_{S N}$ such that (4.1) has exactly two hyperbolic crossing periodic orbits for $b_{S N}<b<0$, only one crossing periodic orbit which is semistable for $b=b_{S N}$, and no crossing periodic orbits for $b<b_{S N}$.

(b4) If $\gamma_{R}+\gamma_{L}>0$, then there is always an unstable crossing periodic orbit. If in addition $a_{R} a_{L}=0$, then that crossing periodic orbit is unique.

In Figure 2, we sketch a global $\left(\gamma_{R}, b\right)$-bifurcation set for the generic case $\gamma_{L}<0$ and $a_{R}<0<a_{L}<-a_{R}$, according to Theorems 4.3, 4.5, and 4.6. Under these conditions, there are no real equilibrium points and only one pseudonode in $\Sigma^{s}$ for $b \neq 0$, which becomes a pseudofocus for $b=0$. Some different bifurcation lines appear in the quoted figure. At the horizontal axis $b=0$, the sliding set becomes the origin; from Proposition 4.2, the pseudofocus at the origin is asymptotically stable for $\gamma_{R}<\gamma_{A}$, being unstable for $\gamma_{R} \geqslant \gamma_{A}$, where

$$
\gamma_{A}=\frac{a_{R} \gamma_{L}}{a_{L}}>-\gamma_{L}>0 .
$$

For $b \neq 0$, the sliding set $\Sigma^{s}$ is a segment with two invisible tangencies at its endpoints: the origin and the point $(0, b)$. Also, there appears a pseudonode in $\Sigma^{s}$, which is stable for $b<0$ and unstable when $b>0$. Thus, in passing from a situation with $b \neq 0$ to the case $b=0$, we have a collision of two invisible tangencies. Such a collision is in fact the bifurcation phenomenon called $I_{2}$ or pseudo-Hopf bifurcation in [13]. Such pseudo-Hopf bifurcation $(p-\mathrm{H}$

Copyright $\odot$ by SIAM. Unauthorized reproduction of this article is prohibited. 


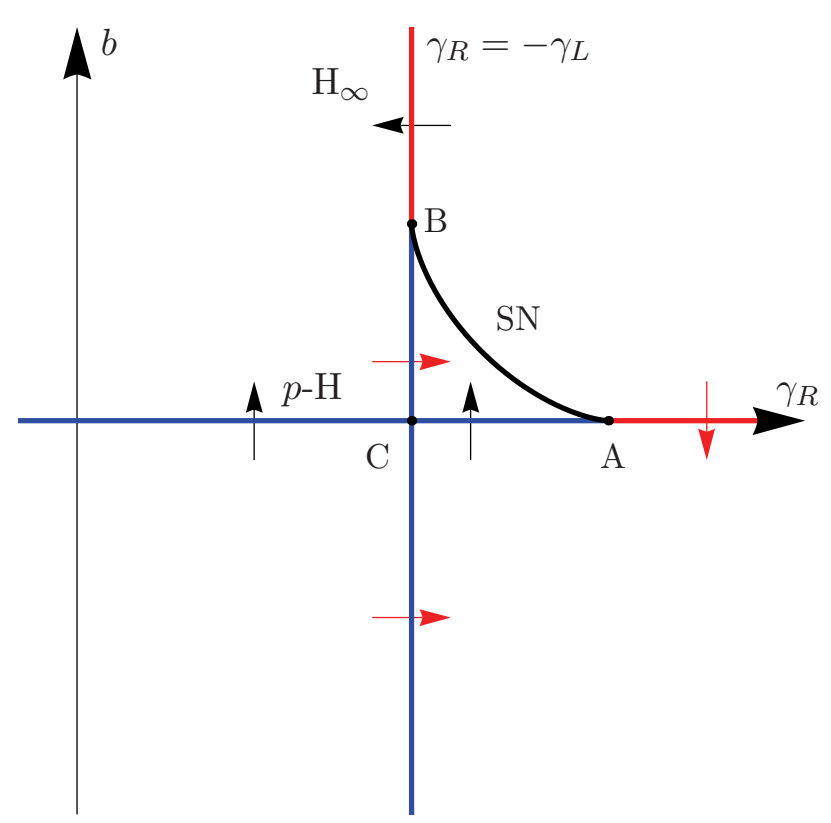

Figure 2. Bifurcation set in the plane $\left(\gamma_{R}, b\right)$ for the case $\gamma_{L}<0$ and $a_{R}<0<a_{L}<-a_{R}$, that is, $\gamma_{A}>-\gamma_{L}$. Different bifurcation curves appear: $H_{\infty}$ stands for Hopf at infinity, SN indicates saddle-node of periodic orbits, and $p$-H means pseudo-Hopf. There are also two codimension-two bifurcation points $A=\left(\gamma_{A}, 0\right)$, $B=\left(-\gamma_{L}, b_{\infty}\right)$ and another $C=\left(-\gamma_{L}, 0\right)$ where two different bifurcations (one local and and another global) simultaneously appear.

in Figure 2) is supercritical for $\gamma_{R}<\gamma_{A}$ and subcritical for $\gamma_{R}>\gamma_{A}$. This is indicated in the figure with an arrow that defines the birth of a crossing limit cycle, black for the stable case and red for the unstable one. Thus, in the plane $\left(\gamma_{R}, b\right)$ the point $A=\left(\gamma_{A}, 0\right)$ represents a degenerate bifurcation point where the character of the bifurcation changes.

Consider a small neighborhood of point $A$ in Figure 2. For $b<0$ we find one crossing stable limit cycle (Theorem 4.6(b4)); for $b>0$ we have two crossing limit cycles for $\gamma_{R}<\gamma_{A}$ (Theorem 4.5(b3)) and no crossing limit cycles when $\gamma_{R}>\gamma_{A}$ (Theorem 4.5(b1)). However, for $\gamma_{R}<\gamma_{A}$ and $b$ sufficiently big, Theorem 4.5(b3) tells us that there are no crossing limit cycles. In fact, it can be shown that, in the quadrant $\gamma_{R}<\gamma_{A}$ and $b>0$, a bifurcation curve SN emanates from the point $A$, corresponding to the collapse of two crossing periodic orbits with different stability, disappearing in the collision, as in degenerate Hopf bifurcations for smooth systems. A similar codimension-two bifurcation for piecewise smooth systems is called invisible fold-fold singularity in [6], where a piecewise polynomial normal form is introduced to obtain the same local bifurcation behavior. From our results, it seems possible to obtain the generic unfolding of such a singularity by using only piecewise linear normal forms; this is an important problem worth further study.

Regarding the straight line $\gamma_{R}=-\gamma_{L}$, we see that for $b<b_{\infty}$ there appears a subcritical Hopf bifurcation at infinity, $\mathrm{H}_{\infty}$, leading to the birth of an unstable crossing periodic orbit of big amplitude for $\gamma_{R}>-\gamma_{L}$. Analogously, for $b>b_{\infty}$ there appears a supercritical Hopf bifurcation at infinity, leading to the birth of a stable crossing periodic orbit of big amplitude 


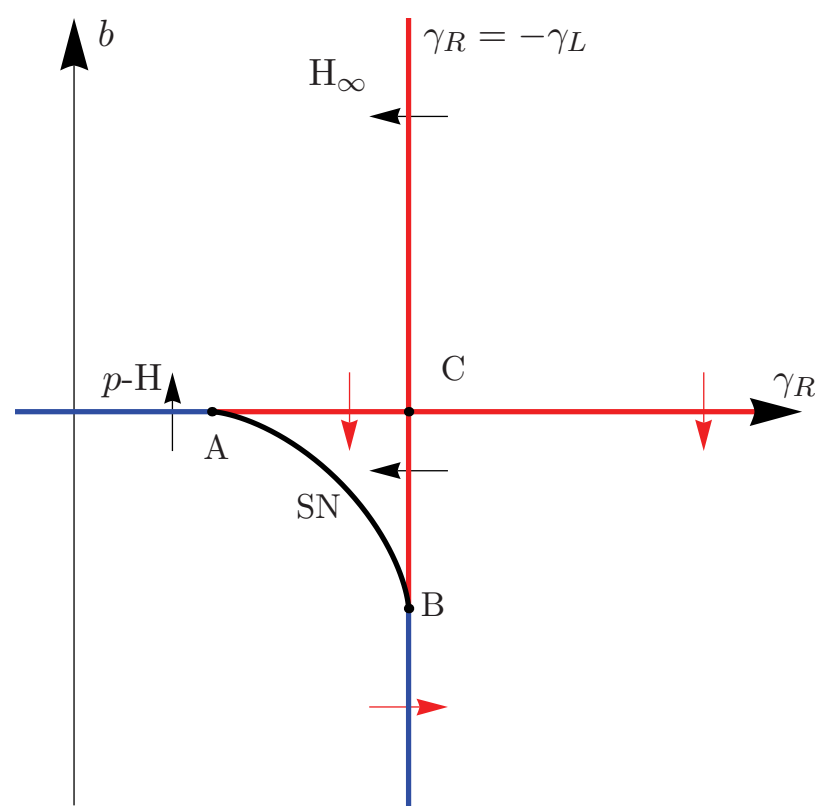

Figure 3. Bifurcation set in the plane $\left(\gamma_{R}, b\right)$ for the case $\gamma_{L}<0$ and $-a_{L}<a_{R}<0<a_{L}$, that is, $\gamma_{A}<-\gamma_{L}$. Labels have the same meaning as in previous figure.

for $\gamma_{R}<-\gamma_{L}$. As before, in the plane $\left(\gamma_{R}, b\right)$, at the point $B=\left(-\gamma_{L}, b_{\infty}\right)$ we have a degenerate bifurcation point similar to the degenerate point $A$ of the previous paragraph, where another curve of bifurcation points corresponding to the collision of two limit cycles begins. The complete analysis of this Hopf bifurcation at infinity and its possible degeneracies will appear elsewhere.

We conjecture that the SN curve joins the points $A$ and $B$, so that the triangle-shaped region defined by such a curve is the parameter locus where two crossing periodic orbits coexist. We also conjecture the uniqueness of the crossing periodic orbit in the region defined by $\gamma_{L} \gamma_{R}<0$ and $b\left(\gamma_{L}+\gamma_{R}\right)<0$, which might be shown by adequately extending the results in [15]. We would like to remark that, regarding Figure 2, our results show the nonexistence of crossing periodic orbits in the quadrant with $b\left(\gamma_{R}+\gamma_{L}\right)>0$ and the uniqueness of the crossing periodic orbit in the quadrant $\gamma_{R} \gamma_{L} \geqslant 0$ with $b\left(\gamma_{R}+\gamma_{L}\right)<0$.

For the sake of completeness, we sketch in Figure 3 the bifurcation set for the case $\gamma_{L}<0$ and $a_{R}<0<a_{L}<-a_{R}$. Here we have $b_{\infty}<0$ and

$$
0<\gamma_{A}=\frac{a_{R} \gamma_{L}}{a_{L}}<-\gamma_{L}
$$

Finally, we note that when $a_{L}=-a_{R}$ we have $\gamma_{A}=-\gamma_{L}$ and $b_{\infty}=0$. So the two degenerate Hopf bifurcation points collide at the point $C=\left(-\gamma_{L}, 0\right)$ in the plane $\left(\gamma_{R}, b\right)$, and then the bifurcation set can be obtained from either Figure 2 or Figure 3 by eliminating the triangle-shaped region. This common point then corresponds to the reversible nonlinear global center predicted in Proposition 4.2(b). 
5. Examples. Next, we illustrate the usefulness of the previous results by considering their application to some DPWL systems; namely, we consider a model regarding the direct voltage control of an electronic Buck converter under idealized switching, and the MorrisLecar model of neural activity in one cell. For the sake of simplicity we will represent the discontinuity by using the Heaviside step function $h(\cdot)$; namely,

$$
h(z)= \begin{cases}0 & \text { if } z<0 \\ 1 & \text { if } z>0\end{cases}
$$

5.1. Discontinuous control in the Buck electronic converter. Our first example comes from power electronics and corresponds to the direct voltage control of the Buck converter under idealized switching. Its dimensionless equations are (see $[4,16])$

$$
\left(\begin{array}{c}
\dot{X} \\
\dot{Y}
\end{array}\right)=\left(\begin{array}{cc}
-\gamma_{1} & 1 \\
-1 & -\gamma_{2}
\end{array}\right)\left(\begin{array}{c}
X \\
Y
\end{array}\right)+h\left(X_{r e f}-X\right)\left(\begin{array}{c}
0 \\
1
\end{array}\right)
$$

where $\gamma_{1}>0, \gamma_{2}>0$ are normalized parameters of the circuit, $X, Y$ are the normalized load voltage and impedance current, and the desired normalized voltage in the load $X_{r e f}$ fulfills the condition

$$
0<X_{r e f}<\frac{1}{1+\gamma_{1} \gamma_{2}}<1
$$

By setting $x=X-X_{r e f}, y=Y$, we get

$$
\left(\begin{array}{l}
\dot{x} \\
\dot{y}
\end{array}\right)=\left(\begin{array}{cc}
-\gamma_{1} & 1 \\
-1 & -\gamma_{2}
\end{array}\right)\left(\begin{array}{l}
x \\
y
\end{array}\right)-\left(\begin{array}{c}
\gamma_{1} X_{r e f} \\
X_{r e f}-1+h(x)
\end{array}\right) \text {. }
$$

Then $T=T^{-}=T^{+}=-\gamma_{1}-\gamma_{2}, D=D^{-}=D^{+}=1+\gamma_{1} \gamma_{2}$, and after applying Proposition 3.1 , we get

$$
\begin{gathered}
\left(\begin{array}{l}
\dot{x} \\
\dot{y}
\end{array}\right)=\left(\begin{array}{rr}
T & -1 \\
D & 0
\end{array}\right)\left(\begin{array}{l}
x \\
y
\end{array}\right)-\left(\begin{array}{c}
0 \\
1-D \cdot X_{r e f}
\end{array}\right) \quad \text { if } x<0, \\
\left(\begin{array}{c}
\dot{x} \\
\dot{y}
\end{array}\right)=\left(\begin{array}{rr}
T & -1 \\
D & 0
\end{array}\right)\left(\begin{array}{l}
x \\
y
\end{array}\right)-\left(\begin{array}{c}
0 \\
-D \cdot X_{\text {ref }}
\end{array}\right) \quad \text { if } x>0 .
\end{gathered}
$$

Now, it is easy to see that $b=0$ (the sliding set shrinks to the origin), $a^{-}=1-D \cdot X_{\text {ref }}>0$ (see (5.1)), and $a^{+}=-D \cdot X_{\text {ref }}<0$, so that we have no inner equilibria in the linearity regions. From Proposition 3.7 we conclude that there are no crossing periodic orbits. Typically, the condition $\left|\gamma_{1}-\gamma_{2}\right|<2$ holds, which implies $T^{2}-4 D<0$, and so the dynamics is of focus-focus type. Then in (4.1) we have $\gamma_{L}=\gamma_{R}<0$ and $a_{R}<0<a_{L}$, so that from Proposition 4.2(a) we deduce that the pseudoequilibrium point is stable. Therefore, from Corollary 4.4 we see that the origin is globally asymptotically stable, which is the goal of the designed discontinuous control strategy. 
5.2. A PWL version of the Morris-Lecar model. In [19], a preliminary study of a PWL version of the Morris-Lecar model for neural activity is presented. We will see that it is a particular instance of system (1.1). We recall that the equations of the PWL version of the Morris-Lecar model are

$$
\begin{aligned}
& \frac{d v}{d t}=-\frac{v}{\tau}-w+\mu h(v-\vartheta)+I, \\
& \frac{d w}{d t}=\delta[\beta v-\gamma w+\alpha h(v-\vartheta)],
\end{aligned}
$$

where the state variables $v$ and $w$ stand for the action potential variable and the recovery variable, $h(\cdot)$ is the Heaviside function, and we assume that all the parameters $\alpha, \beta, \gamma, \delta, \tau, \vartheta$ are positive; see [19] again for the meaning of different parameters. This system can be written in the form (1.1) by the linear change of variables $x=v-\vartheta, y=w$, and then we get

$$
\dot{\mathbf{x}}=\left(\begin{array}{cc}
-\tau^{-1} & -1 \\
\delta \beta & -\delta \gamma
\end{array}\right) \mathbf{x}+\left(\begin{array}{c}
I-\vartheta \tau^{-1}+\mu h(x) \\
\delta \beta \vartheta+\delta \alpha h(x)
\end{array}\right)
$$

Next, we arrive at the canonical form of Proposition 3.1 with

$$
T=T^{-}=T^{+}=-\tau^{-1}-\delta \gamma, \quad D=D^{-}=D^{+}=\delta\left(\beta+\gamma \tau^{-1}\right),
$$

the discontinuity parameter $b=\mu>0$, and

$$
a^{-}=\delta \gamma\left(I-\vartheta\left(\frac{1}{\tau}+\frac{\beta}{\gamma}\right)\right), \quad a^{+}=\delta \gamma\left(I-\vartheta\left(\frac{1}{\tau}+\frac{\beta}{\gamma}\right)+\mu-\frac{\alpha}{\gamma}\right) .
$$

Here, we look only for the cases studied in this paper. The dynamics of this model is of a focus-focus type when $T^{2}-4 D<0$, that is,

$$
\beta>\frac{\left(\tau^{-1}-\delta \gamma\right)^{2}}{4 \delta}
$$

and the condition for nonexistence of inner equilibria in the linearity regions, namely $a^{+} \leqslant$ $0 \leqslant a^{-}$, translates to

$$
\vartheta\left(\frac{1}{\tau}+\frac{\beta}{\gamma}\right) \leqslant I \leqslant \vartheta\left(\frac{1}{\tau}+\frac{\beta}{\gamma}\right)+\frac{\alpha}{\gamma}-\mu
$$

Hence for the particular set of parameters defined by (5.2) and (5.3), which is nonempty for $\alpha>\gamma \mu$, the dynamical behavior of the PWL Morris-Lecar model can be described by means of Theorem 4.5. Since both traces are negative, then the case (a) of the quoted theorem applies, and we conclude that under these assumptions there is only one crossing limit cycle and it is stable, being responsible for the asymptotic periodic dynamics observed in such a PWL Morris-Lecar model. 
6. Conclusions. The study of all possible dynamics in discontinuous PWL systems with two zones is by no means a trivial task, involving a large number of parameters. In this paper, some headway is made in addressing this problem by proposing a canonical form with fewer parameters. Nevertheless, the full rigorous analysis of this simpler family remains a formidable challenge, this paper being only the first of a series to complete the task.

The existing topological equivalence between the dynamics not involving sliding orbits of a given system and that of its reduced form has been exploited for the study of crossing periodic orbits. As a first result, a useful necessary condition for existence of such periodic orbits has been derived.

We have limited our study to DPWL systems whose dynamics rotates around the sliding set, resembling that of a smooth focus. Here, the case where there are no real equilibrium points, describing the possible dynamical behavior both in the phase portrait and parameter space, has been sorted out. Even for this restricted case, the usefulness of the followed approach is emphasized with a couple of applications.

Certain bifurcation sets show different codimension-two Hopf bifurcation points that deserve further study. The existence of up to two limit cycles surrounding the whole sliding set has been proved here. The remaining cases with real foci, where up to three nested crossing limit cycles are possible, are left for a forthcoming work. It is an open problem to determine whether three is the maximum number of nested limit cycles.

The proposed canonical form possesses a sliding set which is controlled by a single parameter. Even its sliding dynamics is in general not topologically equivalent to the corresponding sliding dynamics of the original system; the canonical form seems to be capable of reproducing most of the generic bifurcation phenomena to be found in Filippov systems. Thus, the PWL form obtained could be a valuable tool for obtaining a generic unfolding of some singularities of high codimension.

7. Proof of main results. In this section, we give the proofs of the results included in section 4. We begin by considering some Poincaré maps that we introduce in order to look for crossing periodic orbits.

7.1. Analysis of the Poincaré map. We start by giving the expression of the solutions in each linearity zone. For $x \neq 0$, system (4.1) can be written as

$$
\dot{\mathbf{x}}=\left(\begin{array}{cr}
2 \gamma & -1 \\
1+\gamma^{2} & 0
\end{array}\right) \mathbf{x}-\left(\begin{array}{c}
-\sigma \\
\rho
\end{array}\right)
$$

for appropriate values of parameters $\gamma, \sigma$, and $\rho$. Its solutions have the form

$$
\left(\begin{array}{c}
x(t)-\bar{x} \\
y(t)-\bar{y}
\end{array}\right)=e^{\gamma t}\left(\begin{array}{cc}
\cos t+\gamma \sin t & -\sin t \\
\left(1+\gamma^{2}\right) \sin t & \cos t-\gamma \sin t
\end{array}\right)\left(\begin{array}{c}
x(0)-\bar{x} \\
y(0)-\bar{y}
\end{array}\right),
$$

where the coordinates $(\bar{x}, \bar{y})$ of the equilibrium point are

$$
\bar{x}=\frac{\rho}{1+\gamma^{2}}, \quad \bar{y}=\frac{2 \gamma \rho}{1+\gamma^{2}}+\sigma .
$$

Copyright $\odot$ by SIAM. Unauthorized reproduction of this article is prohibited. 


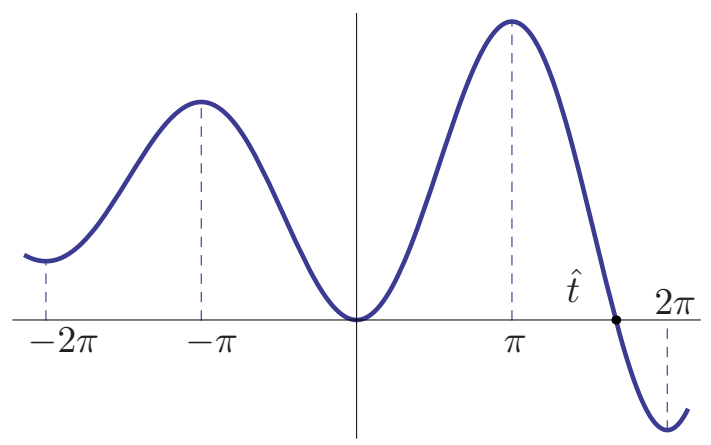

Figure 4. The graph of function $\varphi_{\gamma}(\cdot)$ for a positive value of $\gamma$.

We resort to the auxiliary function $\varphi_{\gamma}(t)=1-e^{\gamma t}(\cos t-\gamma \sin t)$. This function was introduced in [1] and has the symmetry properties

$$
\varphi_{-\gamma}(-t)=\varphi_{\gamma}(t), \quad \varphi_{-\gamma}(t)=\varphi_{\gamma}(-t), \quad \forall \gamma, t \in \mathbb{R},
$$

and the graph shown in Figure 4. Note that function $\varphi_{\gamma}$ has relative maxima at $t= \pm \pi$; when $\gamma>0$, we denote as $\hat{t} \in(\pi, 2 \pi)$ the minimum positive value where the function vanishes.

For system (4.1), the direction of the flow at the line $x=0$ assures that orbits starting at points $\left(0, y_{0}\right)$ with $y_{0} \geqslant 0$ go into the zone $S^{-}$until they reach $\Sigma$ at a point $\left(0, y_{1}\right)$ with $y_{1} \leqslant 0$ after a time $t^{-}$. Thus, we define a left Poincaré map $P_{L}$ as $y_{1}=P_{L}\left(y_{0}\right)$, with $P_{L}(0)=0$.

To determine $P_{L}$ we set $\sigma=0$ and $\rho=a_{L} \geqslant 0$ in (7.2) and consider different cases. If $\gamma_{L}=0$, then

$$
P_{L}\left(y_{0}\right)=-y_{0}, \quad y_{0} \geqslant 0 .
$$

If $a_{L}=0$, then the origin is a boundary equilibrium point. From (7.2) we obtain $t^{-}=\pi$, and

$$
y_{1}=P_{L}\left(y_{0}\right)=-e^{\gamma_{L} \pi} y_{0}, \quad y_{0} \geqslant 0 .
$$

If $a_{L}>0$, then the origin is a left invisible tangency point. By solving (7.2), we obtain the parametric representation of the left Poincaré map,

$$
y_{0}=\frac{a_{L}}{1+\gamma_{L}^{2}} \frac{e^{-\gamma_{L} t^{-}} \varphi_{\gamma_{L}}\left(t^{-}\right)}{\sin t^{-}}, \quad y_{1}=-\frac{a_{L}}{1+\gamma_{L}^{2}} \frac{e^{\gamma_{L} t^{-}} \varphi_{-\gamma_{L}}\left(t^{-}\right)}{\sin t^{-}}
$$

Direct computations from (7.5) show that the two first derivatives of the left Poincaré map when $a_{L} \gamma_{L} \neq 0$ are given by

$$
\begin{gathered}
P_{L}^{\prime}\left(y_{0}\right)=-\frac{\varphi_{\gamma_{L}}\left(t^{-}\right)}{\varphi_{-\gamma_{L}}\left(t^{-}\right)}=\frac{y_{0}}{P_{L}\left(y_{0}\right)} e^{2 \gamma_{L} t^{-}}<0 \\
P_{L}^{\prime \prime}\left(y_{0}\right)=\frac{2 a_{L}^{2}}{1+\gamma_{L}^{2}} \frac{\sinh \gamma_{L} t^{-}-\gamma_{L} \sin t^{-}}{P_{L}^{3}\left(y_{0}\right)} e^{3 \gamma_{L} t^{-}} .
\end{gathered}
$$

Copyright ( $\odot$ by SIAM. Unauthorized reproduction of this article is prohibited. 
Integrating the system in the zone $S^{+}$from the point $\left(0, z_{0}\right)$ with $z_{0} \leqslant b$ big enough, after a time $t^{+}$we arrive at the point $\left(0, z_{1}\right)$, with $z_{1} \geqslant b$. So we can define the right Poincaré map $P_{R}$ as $z_{1}=P_{R}\left(z_{0}\right)$ with $P_{R}(b)=b$.

In the trivial case $\gamma_{R}=0$, from (7.2) we explicitly obtain

$$
z_{1}=P_{R}\left(z_{0}\right)=-z_{0}+2 b, \quad z_{0} \leqslant b .
$$

If $a_{R}=0$, then the point $(0, b)$ is a boundary equilibrium point, and from (7.2) we obtain $t^{+}=\pi$ and

$$
z_{1}=P_{R}\left(z_{0}\right)=-e^{\gamma_{R} \pi} z_{0}+\left(1+e^{\gamma_{R} \pi}\right) b, \quad z_{0} \leqslant b .
$$

If $a_{R}<0$, the point $(0, b)$ is a right invisible tangency point, and the parametric representation of the right Poincaré map is given by

$$
z_{0}=b+\frac{a_{R}}{1+\gamma_{R}^{2}} \frac{e^{-\gamma_{R} t^{+}} \varphi_{\gamma_{R}}\left(t^{+}\right)}{\sin t^{+}}, \quad z_{1}=b-\frac{a_{R}}{1+\gamma_{R}^{2}} \frac{e^{\gamma_{R} t^{+}} \varphi_{-\gamma_{R}}\left(t^{+}\right)}{\sin t^{+}} .
$$

The two first derivatives of $P_{R}$ when $a_{R} \gamma_{R} \neq 0$ are given by

$$
\begin{gathered}
P_{R}^{\prime}\left(z_{0}\right)=\frac{z_{0}-b}{P_{R}\left(z_{0}\right)-b} e^{2 \gamma_{R} t^{+}}<0, \\
P_{R}^{\prime \prime}\left(z_{0}\right)=\frac{2 a_{R}^{2}}{1+\gamma_{R}^{2}} \frac{\sinh \gamma_{R} t^{+}-\gamma_{R} \sin t^{+}}{\left(P_{R}\left(z_{0}\right)-b\right)^{3}} e^{3 \gamma_{R} t^{+}} .
\end{gathered}
$$

Finally, as the main tool in looking for crossing periodic orbits, we define the Poincaré map $P$ as the composition $P=P_{R} \circ P_{L}$. Obviously, the first and second derivatives of map $P$ are

$$
P^{\prime}=\left(P_{R}^{\prime} \circ P_{L}\right) \cdot P_{L}^{\prime}, \quad P^{\prime \prime}=\left(P_{R}^{\prime \prime} \circ P_{L}\right)\left(P_{L}^{\prime}\right)^{2}+\left(P_{R}^{\prime} \circ P_{L}\right) \cdot P_{L}^{\prime \prime} .
$$

Recalling that both parameters $t^{+}$and $t^{-}$(see (7.5) and (7.10)) belong to the interval $(0, \pi)$, we summarize some properties of maps $P_{L}, P_{R}$, and $P$.

Proposition 7.1. Assume that $a_{L} \geqslant 0$ in system (4.1). Then the following statements hold:

(a) If $a_{L} \gamma_{L}=0$, then $P_{L}(y)=-e^{\gamma_{L} \pi} y$ for all $y \geqslant 0$.

(b) If $a_{L}>0$, then the left Poincaré map $P_{L}$ given in (7.5) is well defined for all $y \geqslant 0$, with $t \in(0, \pi)$; in particular we have $P_{L}(0)=0$. Its first four derivatives at the point $y=0$ are

$$
\begin{aligned}
& P_{L}^{\prime}(0)=-1, \quad P_{L}^{\prime \prime}(0)=-\frac{8 \gamma_{L}}{3 a_{L}} \\
& P_{L}^{\prime \prime \prime}(0)=-\frac{32 \gamma_{L}^{2}}{3 a_{L}^{2}}, \quad P_{L}^{I V}(0)=-32 \gamma_{L} \frac{79 \gamma_{L}^{2}-9}{45 a_{L}^{3}}
\end{aligned}
$$

(c) If $a_{L}>0$ and $\gamma_{L} \neq 0$, then for all $y \geqslant 0, P_{L}^{\prime}(y)<0$,

$$
\lim _{y \rightarrow 0^{+}} P_{L}^{\prime}(y)=-1, \quad \lim _{y \rightarrow \infty} P_{L}^{\prime}(y)=-e^{\gamma_{L} \pi}, \quad \operatorname{sign} P_{L}^{\prime \prime}(y)=-\operatorname{sign} \gamma_{L},
$$

Copyright (C) by SIAM. Unauthorized reproduction of this article is prohibited. 
and map $P_{L}$ has the asymptote $A_{L}$ given by

$$
A_{L}(y)=-e^{\gamma_{L} \pi} y+\frac{2 a_{L} \gamma_{L}}{1+\gamma_{L}^{2}}\left(1+e^{\gamma_{L} \pi}\right)
$$

with $\operatorname{sign}\left(A_{L}(y)-P_{L}(y)\right)=\operatorname{sign}\left(\gamma_{L}\right)$.

Proof. Statement (a) follows from (7.3) and (7.4).

The first assertion of statement (b) is straightforward. When $a_{L}>0$, the first derivative at the point $y=0$ follows directly from $(7.6)$. Take a point $(0, \varepsilon)$ with $0<\varepsilon \ll 1$, and consider the point $\left(0, P_{L}(\varepsilon)\right)$ in the same orbit after a time $\hat{t}$. We will follow an approach similar to that taken in section 4.19 of [7]. Since the solutions of a linear system are analytical, from (7.1) with $\sigma=0, \rho=a_{L}, \gamma=\gamma_{L}$ and dropping the superscripts for simplicity, one gets for the solutions around an invisible tangency point

$$
\begin{aligned}
& x(t)=-\varepsilon t+\frac{a-2 \gamma \varepsilon}{2} t^{2}+\frac{2 a \gamma+\left(1-3 \gamma^{2}\right) \varepsilon}{6} t^{3}+\frac{a\left(3 \gamma^{2}-1\right)+4 \gamma \varepsilon\left(1-\gamma^{2}\right)}{24} t^{4}+\cdots, \\
& y(t)=\varepsilon-a t+\left(1+\gamma^{2}\right)\left(-\frac{\varepsilon}{2} t^{2}+\frac{a-2 \gamma \varepsilon}{6} t^{3}+\frac{2 a \gamma+\varepsilon\left(1-3 \gamma^{2}\right)}{24} t^{4}+\cdots\right) .
\end{aligned}
$$

Imposing that $x(\hat{t})=0$ with $\hat{t}>0$ and inverting series (7.15), we obtain

$$
\hat{t}=\frac{2}{a} \varepsilon+\frac{4 \gamma}{3 a^{2}} \varepsilon^{2}+\frac{2\left(5 \gamma^{2}-3\right)}{9 a^{3}} \varepsilon^{3}+\frac{8 \gamma\left(17 \gamma^{2}-27\right)}{135 a^{4}} \varepsilon^{4}+\cdots
$$

and so

$$
y(\hat{t})=P_{L}(\varepsilon)=-\varepsilon-\frac{4 \gamma}{3 a} \varepsilon^{2}-\frac{16 \gamma^{2}}{9 a^{2}} \varepsilon^{3}-\frac{4 \gamma\left(79 \gamma^{2}-9\right)}{135 a^{3}} \varepsilon^{4}+\cdots,
$$

concluding the proof of statement (b).

We know that $t^{-} \in(0, \pi)$, and from (7.6) we have

$$
\lim _{y \rightarrow 0^{+}} P_{L}^{\prime}(y)=\lim _{t^{-} \rightarrow 0^{+}} P_{L}^{\prime}(y)=-1, \quad \lim _{y \rightarrow \infty} P_{L}^{\prime}(y)=\lim _{t^{-} \rightarrow \pi} P_{L}^{\prime}(y)=-e^{\gamma_{L} \pi} .
$$

Since $\operatorname{sign}\left[\sinh \gamma_{L} t^{-}-\gamma_{L} \sin t^{-}\right]=\operatorname{sign} \gamma_{L}$, it is easy to see from (7.7) that $\operatorname{sign} P_{L}^{\prime \prime}(y)=$ $-\operatorname{sign} \gamma_{L}$. The computation of the asymptote is straightforward, and statement (c) is shown.

The following proposition about the right Poincaré map is similar and is given without proof.

Proposition 7.2. Assuming that $a_{R} \leqslant 0$ in system (4.1), the following statements hold:

(a) If $a_{R} \gamma_{R}=0$, then $P_{R}(y)=-e^{\gamma_{R} \pi} y+\left(1+e^{\gamma_{R} \pi}\right) b$ for all $y \leqslant b$.

(b) If $a_{R}<0$, then the right Poincaré map $P_{R}$ given in (7.10) is defined for all $y \leqslant b$, where $t \in(0, \pi)$; in particular we have $P_{R}(b)=b$. Its first four derivatives at the point $y=b$ are

$$
\begin{aligned}
& P_{R}^{\prime}(b)=-1, \quad P_{R}^{\prime \prime}(b)=-\frac{8 \gamma_{R}}{3 a_{R}} \\
& P_{R}^{\prime \prime \prime}(b)=-\frac{32 \gamma_{R}^{2}}{3 a_{R}^{2}}, \quad P_{R}^{I V}(b)=-32 \gamma_{R} \frac{79 \gamma_{R}^{2}-9}{45 a_{R}^{3}}
\end{aligned}
$$

Copyright (C) by SIAM. Unauthorized reproduction of this article is prohibited. 

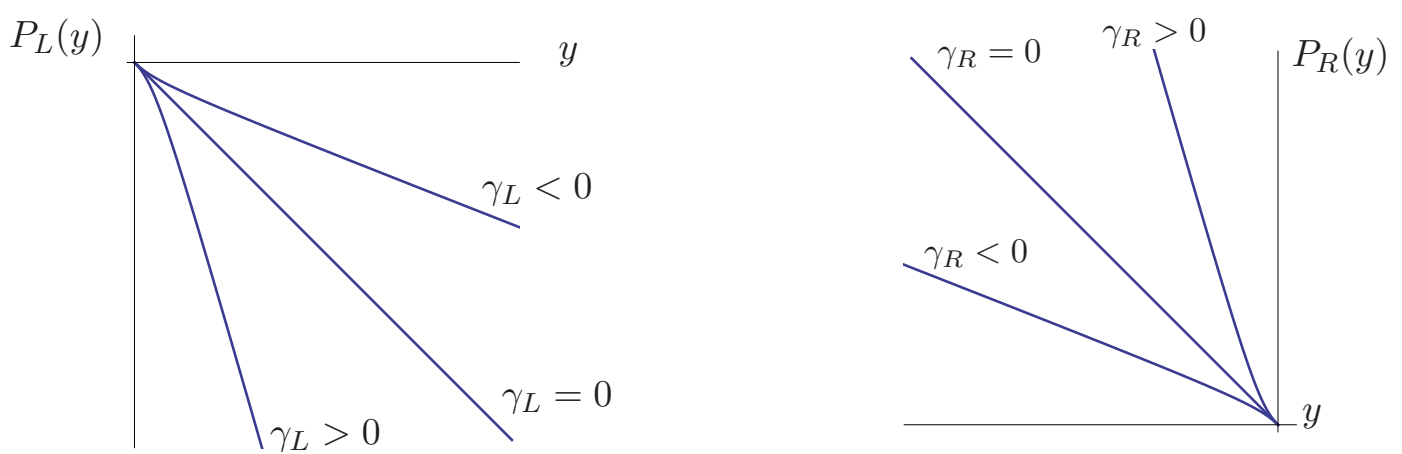

Figure 5. The left Poincaré map for different values of parameter $\gamma_{L}$, and the right Poincaré map for different values of parameter $\gamma_{R}$ and $b=0$.

(c) If $a_{R}<0$ and $\gamma_{R} \neq 0$, then $P_{R}^{\prime}(y)<0$ for all $y \leqslant b$ and

$$
\lim _{y \rightarrow b^{-}} P_{R}^{\prime}(y)=-1, \quad \lim _{y \rightarrow-\infty} P_{R}^{\prime}(y)=-e^{\gamma_{R} \pi}, \quad \operatorname{sign} P_{R}^{\prime \prime}(y)=\operatorname{sign} \gamma_{R} .
$$

(d) Map $P_{R}^{-1}$ has the asymptote $A_{R}^{-1}$ given by

$$
A_{R}^{-1}(y)=-e^{-\gamma^{+} \pi} y+\left(1+e^{-\gamma^{+} \pi}\right)\left(b+\frac{2 a_{R} \gamma_{R}}{1+\gamma_{R}^{2}}\right)
$$

with $\operatorname{sign}\left(P_{R}^{-1}(y)-A_{R}^{-1}(y)\right)=\operatorname{sign}\left(\gamma_{R}\right)$.

In Figure 5, we show different graphs of left and right Poincaré maps depending on the parameters. As a consequence of the above results we can enumerate some properties of the complete Poincaré map.

Proposition 7.3 (properties of the full Poincaré map). Assuming that $a_{R} \leqslant 0 \leqslant a_{L}$ in system (4.1), the following statements hold:

(a) The Poincaré map $P$ is defined for $y \geqslant 0$.

(b) If $a_{L} \gamma_{L}=a_{R} \gamma_{R}=0$, then the Poincaré map $P$ is given by

$$
P(y)=e^{\left(\gamma_{R}+\gamma_{L}\right) \pi} y+\left(1+e^{\gamma_{R} \pi}\right) b .
$$

(c) For all $y \geqslant 0$, the derivative of the Poincaré map is given by

$$
P^{\prime}(y)=\frac{y}{P_{L}(y)} \frac{P_{L}(y)-b}{P(y)-b} e^{2\left(\gamma_{R} t^{+}+\gamma_{L} t^{-}\right)}
$$

and satisfies

$$
\lim _{y \rightarrow \infty} P^{\prime}(y)=e^{\left(\gamma_{R}+\gamma_{L}\right) \pi} .
$$

(d) If $b=0$ and $a_{R}<0<a_{L}$, then the three first derivatives of the Poincaré map at the origin are

$$
P^{\prime}(0)=1, \quad P^{\prime \prime}(0)=\frac{8}{3}\left(\frac{\gamma_{L}}{a_{L}}-\frac{\gamma_{R}}{a_{R}}\right), \quad P^{\prime \prime \prime}(0)=\frac{3}{2}\left(P^{\prime \prime}(0)\right)^{2} .
$$

Copyright (C) by SIAM. Unauthorized reproduction of this article is prohibited. 
When $a_{R} \gamma_{L}=a_{L} \gamma_{R}$, then $P^{\prime \prime}(0)=P^{\prime \prime \prime}(0)=0$, and $P^{I V}(0)$ reduces to

$$
P^{I V}(0)=-\frac{32 \gamma_{R}\left(\gamma_{R}-\gamma_{L}\right)\left(\gamma_{R}+\gamma_{L}\right)}{5 a_{R}^{3} \gamma_{L}^{2}} .
$$

(e) If $a_{R}<0<a_{L}$ and $\gamma_{L}=-\gamma_{R} \neq 0$, then

$$
P_{R}\left[b-\frac{a_{L}}{a_{R}} P_{L}(y)\right]=b-\frac{a_{L}}{a_{R}} y .
$$

If, in addition, $a_{R}=-a_{L}$ and $b=0$, then $P(y)=y$, and we have a nonlinear global center around the origin.

(f) If $a_{R}<0$ and $a_{L}=0$, then $\operatorname{sign} P^{\prime \prime}(y)=\operatorname{sign} \gamma_{R}$. If, in addition, $b=0$, we get $P^{\prime}(0)=e^{\gamma_{L} \pi}$.

(g) If $a_{R}=0$ and $a_{L}>0$, then $\operatorname{sign} P^{\prime \prime}(y)=\operatorname{sign} \gamma_{L}$. If, in addition, $b=0$, we get $P^{\prime}(0)=e^{\gamma_{R} \pi}$.

Proof. Statement (a) directly follows from Propositions 7.1 and 7.2.

If $a_{L} \gamma_{L}=0$, then from (7.3) and (7.4) we have $P_{L}(y) \leqslant 0$ for $y \geqslant 0$, and when $a_{R} \gamma_{R}=0$, from (7.8) and (7.9) we explicitly obtain $P_{R}$. Then by doing the composition $P=P_{R} \circ P_{L}$, statement (b) follows.

From Propositions 7.1 and 7.2 and the chain rule, the statements about the derivatives of the Poincaré map can be easily deduced.

If $\gamma=\gamma_{L}=-\gamma_{R}$, then from (7.5) and (7.10), we get

$$
b-\frac{a_{L}}{a_{R}} P_{L}(y)=P_{R}^{-1}\left(b-\frac{a_{L}}{a_{R}} y\right),
$$

and (7.19) follows. If additionally $a_{R}=-a_{L}$ and $b=0$, it is evident that $P_{R}\left(P_{L}(y)\right)=y$, and statement (e) is proven.

Statement (f) follows from Proposition 7.1(b), Proposition 7.2(c), and the chain rule, taking into consideration that $P_{L}^{\prime \prime}(y)=0$.

Statement (g) follows from Proposition 7.1(c), Proposition 7.2(b), and the chain rule, taking into consideration that $P_{R}^{\prime \prime}(y)=0$.

Remark 7.4. We remark that when $a_{L}<0<a_{R}$, we have $\operatorname{sign} P^{\prime \prime}(0)=\operatorname{sign}\left(a_{L} \gamma_{R}-a_{R} \gamma_{L}\right)$. Moreover, since $\gamma_{R}\left(\gamma_{R}-\gamma_{L}\right)>0$, when $\gamma_{R} \gamma_{L}<0$ we deduce that $\operatorname{sign} P^{I V}(0)=\operatorname{sign}\left(\gamma_{R}+\gamma_{L}\right)$ for $a_{L} \gamma_{R}=a_{R} \gamma_{L}$ and $\gamma_{R} \gamma_{L}<0$.

7.2. Proof of results related to crossing periodic orbits. We start by giving a proof of Proposition 3.7.

Proof of Proposition 3.7. We start by considering the vector field

$$
\mathbf{F}^{\perp}(\mathbf{x})= \begin{cases}\left(-D^{-} x+a^{-}, T^{-} x-y\right)^{T}, & \mathbf{x} \in S^{-}, \\ \left(-D^{+} x+a^{+}, T^{+} x-y+b\right)^{T}, & \mathbf{x} \in S^{+},\end{cases}
$$

which is a field orthogonal to the one defined by (3.4). The Green's formula produces

$$
\oint_{\Gamma^{-} \cup L^{-}} \mathbf{F}^{\perp} d \mathbf{r}=\iint_{\Omega^{-}} T^{-} d \sigma=T^{-} \sigma^{-},
$$

Copyright $\odot$ by SIAM. Unauthorized reproduction of this article is prohibited. 
Table 1

Symmetries of canonical form (4.1).

\begin{tabular}{|c|c|c|}
\hline$\Pi_{1}$ & $\Pi_{2}$ & $\Pi_{3}$ \\
\hline$x \rightarrow x$ & $x \rightarrow-x$ & $x \rightarrow-x$ \\
\hline$y \rightarrow-y$ & $y \rightarrow y+b$ & $y \rightarrow-y-b$ \\
\hline$t \rightarrow-t$ & $t \rightarrow-t$ & $t \rightarrow t$ \\
\hline$\gamma_{L} \rightarrow-\gamma_{L}$ & $\gamma_{L} \rightarrow-\gamma_{R}$ & $\gamma_{L} \rightarrow \gamma_{R}$ \\
\hline$\gamma_{R} \rightarrow-\gamma_{R}$ & $\gamma_{R} \rightarrow-\gamma_{L}$ & $\gamma_{R} \rightarrow \gamma_{L}$ \\
\hline$a_{L} \rightarrow a_{L}$ & $a_{L} \rightarrow-a_{R}$ & $a_{L} \rightarrow-a_{R}$ \\
\hline$b \rightarrow-b$ & $b \rightarrow-b$ & $b \rightarrow b$ \\
\hline$a_{R} \rightarrow a_{R}$ & $a_{R} \rightarrow-a_{L}$ & $a_{R} \rightarrow-a_{L}$ \\
\hline
\end{tabular}

where the continuous extension of the vector field in $S^{-}$to the boundary $\Sigma$ must be considered in $\mathrm{L}^{-}$. Similarly, we have

$$
\oint_{\Gamma^{+} \cup L^{+}} \mathbf{F}^{\perp} d \mathbf{r}=\iint_{\Omega^{+}} T^{+} d \sigma=T^{+} \sigma^{+},
$$

where the continuous extension of the vector field in $S^{+}$to the boundary $\Sigma$ must be considered in $\mathrm{E}^{+}$. By the orthogonality and the above remarks, we have

$$
\oint_{\Gamma^{-} \cup L^{-}} \mathbf{F}^{\perp} d \mathbf{r}=\int_{L^{-}} \mathbf{F}^{\perp} d \mathbf{r}=\int_{y_{L}}^{y_{L}+h}(-y) d y
$$

and

$$
\oint_{\Gamma^{+} \cup L^{+}} \mathbf{F}^{\perp} d \mathbf{r}=\int_{L^{+}} \mathbf{F}^{\perp} d \mathbf{r}=-\int_{y_{L}}^{y_{L}+h}(-y+b) d y .
$$

Adding (7.20) with (7.21), taking into consideration (7.22) and (7.23), we have

$$
T^{-} \sigma^{-}+T^{+} \sigma^{+}=-\int_{y_{L}}^{y_{L}+h} b d y
$$

and the conclusion follows.

Next note that the canonical form given in (4.1) is invariant under the transformations shown in Table 1, where each transformation is the composition of the other two. Obviously, adding the identity transformation $\Pi_{0}$, we should have an invariant transformation group. We can use the above symmetries to reduce the number of cases to be considered in the different proofs. We recall that when $b \neq 0$, after applying either $\Pi_{1}$ or $\Pi_{2}$, we need only to study, as said before, the case $b>0$. Then, we need to consider only the three cases $a_{R}<0<a_{L}$, $a_{R}=a_{L}=0$ and $a_{R}<0, a_{L}=0$ because the remaining case $a_{R}=0$ and $a_{L}>0$ can be reduced to the last one by means of the symmetry $\Pi_{3}$.

Proof of Proposition 4.2. (a) Assuming $a_{L} \gamma_{R}<a_{R} \gamma_{L}$, we cannot have $a_{L}=a_{R}=0$. If $a_{R}<0<a_{L}$, then $\gamma_{L} / a_{L}-\gamma_{R} / a_{R}<0$, and from Proposition $7.3(\mathrm{~d})$ we have $P^{\prime}(0)=1$ and sign $P^{\prime \prime}(0)<0$. If $a_{R}<0$ and $a_{L}=0$, we must have $\gamma_{L}<0$, and from Proposition 7.3(f) we get $P^{\prime}(0)=e^{\gamma_{L} \pi}<1$. In both cases the origin is stable. The proof of the statement when $a_{L} \gamma_{R}>a_{R} \gamma_{L}$ is reduced to the previous proof by using the symmetry $\Pi_{1}$ of Table 1 .

Copyright (C) by SIAM. Unauthorized reproduction of this article is prohibited. 
(b) If $a_{L} \gamma_{R}=a_{R} \gamma_{L}=0$, then from Proposition 7.3(b) we obtain $P^{\prime}(y)=e^{\left(\gamma_{R}+\gamma_{L}\right) \pi}$. If $a_{L} \gamma_{R}=a_{R} \gamma_{L} \neq 0$, then from the assumptions $a_{R}<0<a_{L}, \gamma_{R} \gamma_{L}<0$ and from Proposition $7.3(\mathrm{~d})$ we get $P^{\prime}(0)=1, P^{\prime \prime}(0)=P^{\prime \prime \prime}(0)=0$, but from Remark 7.4 we have sign $P^{I V}(0)=$ $\operatorname{sign}\left(\gamma_{R}+\gamma_{L}\right)$. In any case the statement follows.

Proof of Theorem 4.3. Through this proof we will extensively use the Poincaré map. First note that $P(0)=0$. If system (4.1) has a crossing periodic orbit, then the Poincaré map has a fixed point $\bar{y}$ with $P(\bar{y})=\bar{y}$, and we conclude, by the mean value theorem, that there exists a point $\xi \in(0, \bar{y})$ with $P^{\prime}(\xi)=1$. In the following, all the different possible cases are considered.

(a) In the cases where $a_{R} \gamma_{L}=a_{L} \gamma_{R}=0$, from Proposition 7.3(b) we obtain $P(y)=y$ for all $y \geqslant 0$, and the existence of a global center follows. Thus, by recalling the leading paragraph of this section, we need only to study the cases with $\gamma_{R}=-\gamma_{L} \neq 0$ and $a_{L}-a_{R}>0$.

(i) Assume that $a_{R}<0<a_{L}$. If $a_{R} \gamma_{L}=a_{L} \gamma_{R}$, then $a_{R}=-a_{L}$, and from Proposition 7.3(e) we have $P(y)=y$ and again a global center. If $a_{R} \gamma_{L} \neq a_{L} \gamma_{R}$, then $a_{R} \neq-a_{L}$, and we write $0<\kappa=-a_{L} / a_{R} \neq 1$. If a point $\bar{y}$ with $P_{R}\left(P_{L}(\bar{y})\right)=\bar{y}$ exists, then from Proposition $7.3(\mathrm{e})$ we have $P_{R}\left[\kappa P_{L}(\bar{y})\right]=\kappa \bar{y}$, and so

$$
P_{L}(\bar{y})=\frac{1}{\kappa} P_{R}^{-1}(\kappa \bar{y})=P_{R}^{-1}(\bar{y}) .
$$

Let us introduce the function $h(y)=P_{R}^{-1}(\kappa y)-\kappa P_{R}^{-1}(y)$. Since $h(0)=h(\bar{y})=0$, there exists a value $\xi \in(0, \bar{y})$ such that

$$
h^{\prime}(\xi)=\kappa\left[\left(P_{R}^{-1}\right)^{\prime}(\kappa \xi)-\left(P_{R}^{-1}\right)^{\prime}(\xi)\right]=0,
$$

but this is impossible because the function $P_{R}^{-1}$ has a monotonic derivative. The last assertion comes easily from Proposition 7.2(c). Hence our system has no crossing periodic orbits.

(ii) Suppose that $a_{R}<0$ and $a_{L}=0$. Then $a_{R} \gamma_{L} \neq a_{L} \gamma_{R}$, and from Proposition 7.3(c) and (f) we get

$$
\lim _{y \rightarrow \infty} P^{\prime}(y)=1, \quad \operatorname{sign} P^{\prime \prime}(y)=\operatorname{sign} \gamma_{R}, \quad P^{\prime}(0)=e^{\gamma_{L} \pi} .
$$

We can assume without loss of generality that $\gamma_{R}<0$ and $\gamma_{L}>0$; otherwise apply symmetry $\Pi_{1}$. Then $P^{\prime}$ is decreasing with $P^{\prime}(y)>1$ always, so there cannot exist $\xi$ with $P^{\prime}(\xi)=1$, and consequently the system has no crossing periodic orbits.

(b) Since system (4.1) is a particular instance of system (3.4) where $T^{+}=2 \gamma_{R}$ and $T^{-}=2 \gamma_{L}$, the statement follows from Remark 3.8.

(c) When $\gamma_{R} \gamma_{L}<0$, then by the symmetries of Table 1 we can assume without loss of generality that $\gamma_{R}+\gamma_{L}<0$. Thus, from Proposition 7.3(c) we have $\lim _{y \rightarrow \infty} P^{\prime}(y)<1$, and we need only study the following three cases:

(i) If $a_{R}<0<a_{L}$, then again by the symmetries we can assume without loss of generality that $\gamma_{L}<0, \gamma_{R}>0$. From Proposition $7.3(\mathrm{~d})$ we get $P^{\prime}(0)=1$ and $\operatorname{sign} P^{\prime \prime}(0)=$ $\operatorname{sign}\left(a_{L} \gamma_{R}-a_{R} \gamma_{L}\right)$. As $P^{\prime}(y)$ is eventually less than 1 , we have $P(y)<y$ for $y$ sufficiently big. When $a_{L} \gamma_{R}=a_{R} \gamma_{L}$, from Remark 7.4 we get $P^{\prime \prime}(0)=P^{\prime \prime \prime}(0)=0$ and $\operatorname{sign} P^{I V}(0)=\operatorname{sign}\left(\gamma_{R}+\gamma_{L}\right)<0$. When $a_{L} \gamma_{R}<a_{R} \gamma_{L}$, we have $P^{\prime \prime}(0)<0$. Thus when 
$a_{L} \gamma_{R} \leqslant a_{R} \gamma_{L}$, the number of periodic orbits, if any, is even. When $a_{L} \gamma_{R}>a_{R} \gamma_{L}$, we conclude the existence of one periodic orbit at least. The assertions on uniqueness and nonexistence of periodic orbits now come directly from Theorem 4 of [15].

(ii) If $a_{L}=a_{R}=0$, then $a_{R} \gamma_{L}=a_{L} \gamma_{R}$, and from Proposition 7.3(b) we have $P(y)=$ $e^{\left(\gamma_{R}+\gamma_{L}\right) \pi} y<y$, and there are no crossing periodic orbits.

(iii) If $a_{R}<0$ and $a_{L}=0$, then obviously $a_{R} \gamma_{L} \neq a_{L} \gamma_{R}$, and we distinguish two cases depending on the sign of $\gamma_{L}$ :

If $\gamma_{L}>0$, then $\gamma_{R}<0$, and from Proposition 7.3(f) we have $P^{\prime}(0)>1$ and $P^{\prime \prime}(y)<0$ for all $y>0$. Hence $P^{\prime}$ is decreasing from $P^{\prime}(0)>1$ to $e^{\left(\gamma_{R}+\gamma_{L}\right) \pi}<1$. Then it is easy to show the existence of only one value $\bar{y}$ with $P(\bar{y})=\bar{y}$. Furthermore, $P^{\prime}(\bar{y})<1$, and the system has only one stable crossing periodic orbit.

If $\gamma_{L}<0$, then $\gamma_{R}>0$, and again from Proposition 7.3(f) we have $P^{\prime}(0)<1, P^{\prime \prime}(y)>0$ for all $y>0$. Hence $P^{\prime}$ is increasing with $P^{\prime}(0)<P^{\prime}(y)<e^{\left(\gamma_{R}+\gamma_{L}\right) \pi}<1$, and there are no crossing periodic orbits.

Now, by using elementary properties of the full Poincaré map, the proof of Corollary 4.4 easily follows.

Proof of Corollary 4.4. If the origin is stable and there are no crossing periodic orbits, it is obvious that $P(y)<y$ for all $y>0$, and we are done.

In the following, the existence of periodic behavior in system (4.1) for $b>0$ is considered. Note that the left Poincaré map does not depend upon parameter $b$, but Poincaré maps $P_{R}$ and $P_{R}^{-1}$ linearly depend on parameter $b$. In fact, by emphasizing the dependence on $b$ in the notation, from (7.9) and (7.10) we see that

$$
P_{R}^{-1}(y ; b)=b+P_{R}^{-1}(y-b ; 0)
$$

that is, if we make the change $y \rightarrow y-b$, we should have a right system with $b=0$ having the same orbit, but vertically translated in the amount $b$. Note that (7.24) implies that if we translate the coordinates of every point $\left(y, P_{R}^{-1}(y, 0)\right)$ belonging to the graph of $P_{R}^{-1}(y ; 0)$ to the point

$$
\left(y+b, P_{R}^{-1}(y, 0)+b\right)=\left(y+b, P_{R}^{-1}(y+b, b)\right),
$$

such a point is in the graph of $P_{R}^{-1}(y ; b)$. In short, the graph of $P_{R}^{-1}(y ; b)$ is just a translation in both coordinates of $P_{R}^{-1}(y ; 0)$ by the amount $b$; see Figure 6 .

If $\bar{y}$ is a fixed point of the Poincaré map $P$, then $P(\bar{y})=P_{R}\left(P_{L}(\bar{y}) ; b\right)=\bar{y}$, and so $P_{L}(\bar{y})=P_{R}^{-1}(\bar{y} ; b)$. Hence the existence of crossing periodic orbits is equivalent to the existence of zeros for the function

$$
\Psi_{b}(y)=P_{R}^{-1}(y ; b)-P_{L}(y)=b+P_{R}^{-1}(y-b ; 0)-P_{L}(y), \quad y \geqslant b,
$$

where we have also used (7.24).

Remark 7.5. We remark that if $\Psi_{b}(y) \neq 0$ for $b \geqslant 0$ and $y \geqslant b$, then $\Psi_{b}(y)(P(y)-y)>0$. Effectively, if $\Psi_{b}(y)>0$, then $P_{R}^{-1}(y ; b)>P_{L}(y)$, and since $P_{R}$ is decreasing, from Proposition 7.3 we get $y<P(y)$. Analogously, when $\Psi_{b}(y)<0$, we get $P(y)-y<0$.

The two first derivatives of function $\Psi_{b}$ with respect to the variable $y$ can be computed

Copyright $\odot$ by SIAM. Unauthorized reproduction of this article is prohibited. 


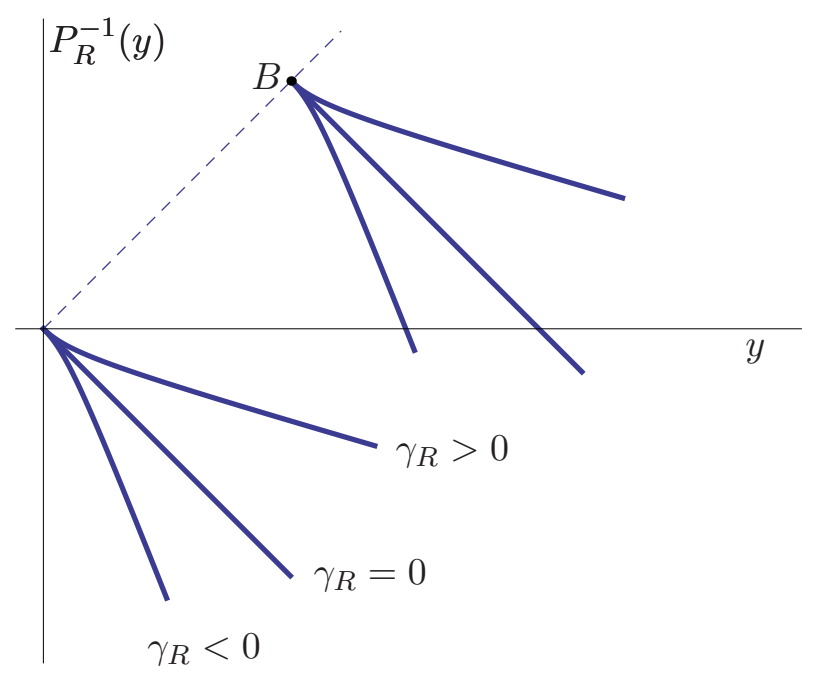

Figure 6. The inverse half Poincaré map $P_{R}^{-1}$ for $b=0$ and several values of parameter $\gamma_{R}$. To obtain the map $P_{R}^{-1}$ for $b>0$, it suffices to translate the graphs to the point $B$, which has the coordinates $(b, b)$.

by using the derivatives of $P_{R}$, which are

$$
\Psi_{b}^{\prime}(y)=\frac{1}{\left(P_{R}^{\prime} \circ P_{R}^{-1}\right)(y ; b)}-P_{L}^{\prime}(y), \quad \Psi_{b}^{\prime \prime}(y)=-\frac{\left(P_{R}^{\prime \prime} \circ P_{R}^{-1}\right)(y ; b)}{\left(P_{R}^{\prime} \circ P_{R}^{-1}\right)^{3}(y ; b)}-P_{L}^{\prime \prime}(y) .
$$

We will derive some properties of function $\Psi_{b}$ with $b>0$, by considering the case $b=0$. Thus, first note that $\Psi_{0}(0)=0$. Next, under the assumptions $a_{R}<0<a_{L}$, from (7.26) and Propositions 7.1(b) and 7.2(b), the first and second derivatives of function $\Psi_{0}$ at the origin are

$$
\Psi_{0}^{\prime}(0)=0, \quad \Psi_{0}^{\prime \prime}(0)=-\frac{8\left(a_{L} \gamma_{R}-a_{R} \gamma_{L}\right)}{3 a_{R} a_{L}} .
$$

Some properties of function $\Psi_{b}$ defined in (7.25) follow.

Lemma 7.6. For system (4.1) with $a_{R} \leqslant 0 \leqslant a_{L}$ and $b>0$, the following statements hold:

(a) If $\Psi_{b}(\bar{y})=0$, then $P(\bar{y})=\bar{y}$, and $\bar{y}$ corresponds to a stable crossing periodic orbit when $\Psi_{b}^{\prime}(\bar{y})<0$ (respectively, unstable for $\Psi_{b}^{\prime}(\bar{y})>0$ ).

(b) Function $\Psi_{b}$ satisfies the inequality $\Psi_{b}(y)>b+\Psi_{0}(y)$ for $y \geqslant b$.

Proof. (a) If $\Psi_{b}(\bar{y})=0$, then it directly follows that $P(\bar{y})=\bar{y}$. Furthermore, as $P_{R}^{-1}(\bar{y})=$ $P_{L}(\bar{y})$, from $(7.26)$ the first derivative of function $\Psi_{b}$ reduces to

$$
\Psi_{b}^{\prime}(\bar{y})=\frac{1}{P_{R}^{\prime}\left(P_{L}(\bar{y})\right)}-P_{L}^{\prime}(y)=\frac{1-P_{R}^{\prime}\left(P_{L}(\bar{y})\right) P_{L}^{\prime}(y)}{P_{R}^{\prime}\left(P_{L}(\bar{y})\right)}=\frac{1-P^{\prime}(\bar{y})}{P_{R}^{\prime}\left(P_{L}(\bar{y})\right)} .
$$

Since $P_{R}$ is decreasing, the above denominator is negative, and the value $\bar{y}$ corresponds to a stable crossing periodic orbit when $\Psi_{b}^{\prime}(\bar{y})<0$ since then $P^{\prime}(\bar{y})<1$. The unstable case follows similarly.

(b) Since map $P_{R}^{-1}$ is decreasing, from (7.24) we have

$$
\Psi_{b}(y)=b+P_{R}^{-1}(y-b ; 0)-P_{L}(y)>b+P_{R}^{-1}(y ; 0)-P_{L}(y)=b+\Psi_{0}(y) .
$$


Lemma 7.7. For system (4.1) with $a_{R} \leqslant 0 \leqslant a_{L}$ the following statements hold:

(a) If $\gamma_{R}+\gamma_{L}=0$, then $\operatorname{sign} \Psi_{0}(y)=\operatorname{sign}\left(a_{L} \gamma_{R}-a_{R} \gamma_{L}\right)$ for $y \geqslant 0$.

(b) If $\gamma_{R}+\gamma_{L} \neq 0, \gamma_{R} \gamma_{L}<0$, and $\left(\gamma_{R}+\gamma_{L}\right)\left(a_{L} \gamma_{R}-a_{R} \gamma_{L}\right) \geqslant 0$, then for $y \geqslant 0$

$$
\operatorname{sign} \Psi_{0}(y)=\left\{\begin{array}{l}
\operatorname{sign}\left(a_{L} \gamma_{R}-a_{R} \gamma_{L}\right) \text { when } a_{L} \gamma_{R} \neq a_{R} \gamma_{L}, \\
\operatorname{sign}\left(\gamma_{R}+\gamma_{L}\right) \text { otherwise. }
\end{array}\right.
$$

Proof. (a) When $\gamma_{R}+\gamma_{L}=0$ and $b=0$, two cases arise. If $a_{L} \gamma_{R}=a_{R} \gamma_{L}$, then from Theorem 4.3(a) we get $P(y)=y$; that is, $\Psi_{0}$ vanishes everywhere. If $a_{L} \gamma_{R} \neq a_{R} \gamma_{L}$, then again from Theorem $4.3\left(\right.$ a) we get $P(y) \neq y$ and $\Psi_{0}(y) \neq 0$ for $y>0$, and from (7.27) the conclusion follows.

(b) From Theorem 4.3(c) we have $P(y) \neq y$ for $y>0$, and from Remark 7.4 we have

$$
\operatorname{sign}(P(y)-y)=\left\{\begin{array}{l}
\operatorname{sign} P^{\prime \prime}(0)=\operatorname{sign}\left(a_{L} \gamma_{R}-a_{R} \gamma_{L}\right) \text { if } a_{L} \gamma_{R} \neq a_{R} \gamma_{L} \\
\operatorname{sign} P^{I V}(0)=\operatorname{sign}\left(\gamma_{R}+\gamma_{L}\right) \text { otherwise, }
\end{array}\right.
$$

and the conclusion follows, taking into account Remark 7.5.

Proof of Theorem 4.5. (a) When $\gamma_{R} \gamma_{L} \geqslant 0$, we distinguish two cases:

(i) If $\gamma_{R}+\gamma_{L} \geqslant 0$, then $\gamma_{R} \geqslant 0, \gamma_{L} \geqslant 0$ and the statement follows from Remark 3.8.

(ii) If $\gamma_{R}+\gamma_{L}<0$, then $\gamma_{R} \leqslant 0$ and $\gamma_{L} \leqslant 0$. From Propositions 7.1 and 7.2 we get $P_{R}^{\prime}(y)<0, P_{R}^{\prime \prime}(y) \leqslant 0, P_{L}^{\prime \prime}(y) \geqslant 0$, for all $y>0$, and so from (7.13) we get $P^{\prime \prime}(y)<0$ always. Since $P(0)=P_{R}(0)>b$ and $\lim _{y \rightarrow \infty} P^{\prime}(y)<1$, from the concavity of map $P$ we conclude the existence of a unique point $\bar{y}$ with $P(\bar{y})=\bar{y}$. Moreover, as $P^{\prime}(\bar{y})<1$, the corresponding crossing periodic orbit is stable.

(b1) Under the assumptions, from Lemma 7.7 we get $\Psi_{0}(y) \geqslant 0$ for all $y \geqslant 0$. Finally, from Lemma 7.6(b) we obtain $\Psi_{b}(y) \geqslant b>0$ for all $y \geqslant b$, and the conclusion follows.

(b2) Under our hypotheses we have $\gamma_{R}=-\gamma_{L} \neq 0$; then we obtain $b_{\infty}>0$. Furthermore, from Proposition 7.3(b) we get $\lim _{y \rightarrow \infty} P^{\prime}(y)=1$. Now, we distinguish two cases:

(i) If $a_{R}<0<a_{L}$, we claim that system (4.1) has at most one crossing periodic orbit. Assume on the contrary that our system has two periodic orbits, so that the Poincare map has two fixed points $\bar{y}_{1}$ and $\bar{y}_{2}$. Then, by defining $\kappa=a_{L} / a_{R}$, the hypotheses enforce $\kappa<-1$, and from (7.19) we get

$$
b-\kappa P_{L}\left(\bar{y}_{i}\right)=b-\kappa P_{R}^{-1}\left(\bar{y}_{i}\right)=P_{R}^{-1}\left(b-\kappa \bar{y}_{i}\right), \quad i=1,2,
$$

where the first equality comes from the obvious fact $P_{L}(\bar{y})=P_{R}^{-1}(\bar{y})$. Let us introduce for $y>b$ the function

$$
h(y)=P_{R}^{-1}(b-\kappa y)-b+\kappa P_{R}^{-1}(y) .
$$

Since $h\left(\bar{y}_{1}\right)=h\left(\bar{y}_{2}\right)=0$, there exists a value $\xi \in\left(\bar{y}_{1}, \bar{y}_{2}\right)$ such that

$$
h^{\prime}(\xi)=-\kappa\left[\left(P_{R}^{-1}\right)^{\prime}(b-\kappa \xi)-\left(P_{R}^{-1}\right)^{\prime}(\xi)\right]=0,
$$

but this is impossible because the function $P_{R}^{-1}$ has a second derivative with constant sign, and so our system has one transversal periodic orbit at most. Without loss of generality we

Copyright (C) by SIAM. Unauthorized reproduction of this article is prohibited. 
can assume $\gamma_{R}=-\gamma_{L}<0$; otherwise, we can apply transformation $\Pi_{1}$ of Table 1. From Propositions 7.1 and 7.2 , we have $P_{L}(y)<A_{L}(y), P_{R}^{-1}(y)<A_{R}^{-1}(y)$, and

$$
A_{R}^{-1}(y)-A_{L}(y)=\left(1+e^{\gamma_{L} \pi}\right)\left(b-b_{\infty}\right) .
$$

When $0<b<b_{\infty}, A_{R}^{-1}(y)<A_{L}(y)$, and from the properties of asymptotes we deduce that for $y$ sufficiently big we must have

$$
P_{R}^{-1}(y)<A_{R}^{-1}(y)<P_{L}(y)<A_{L}(y)
$$

that is, $\Psi_{b}(y)<0$ for $y \gg 1$. Since $\Psi_{b}(b)>0$ the system must have at least one crossing periodic orbit. But we know that there should be only one by the properties of function $h$. Furthermore, this unique periodic orbit is stable because function $\Psi_{b}$ has to be decreasing in a neighborhood of its vanishing value. When $b \geqslant b_{\infty}$, reasoning as before, for $y \gg 1$ we now have

$$
P_{L}(y)<A_{L}(y)<P_{R}^{-1}(y)<A_{R^{-1}}(y) .
$$

Thus $\Psi_{b}(y)>0$ for $y \gg 1$, and system (4.1) might have an even number of periodic orbits or none. Since the system has one transversal periodic orbit at most, we conclude that there are no periodic orbits in this case, and the statement follows.

(ii) If $a_{R}<0$ and $a_{L}=0$, then we must have $\gamma_{R}=-\gamma_{L}>0$. From Propositions 7.1 and 7.2 then $A_{R}^{-1}(y)<P_{R}^{-1}(y)$ and

$$
A_{R}^{-1}(y)-P_{L}(y)=\left(1+e^{\gamma_{L} \pi}\right)\left(b-b_{\infty}\right) .
$$

If $b<b_{\infty}$ and $y \gg 1$, from the properties of asymptotes and the previous equality, we have $A_{R}^{-1}(y)<P_{R}^{-1}(y)<P_{L}(y)$, that is, $\Psi_{b}(y)<0$ for $y \gg 1$, and taking into account that $\Psi_{b}(b)>0$, we conclude the existence of one crossing periodic orbit, at least. From Proposition 7.3(f) we get $P^{\prime \prime}(y)>0$, and so $P^{\prime}(y)<1$ for all $y>0$. Then the Poincaré map has only one fixed point, which corresponds with the only crossing periodic orbit, being stable. However, when $b \geqslant b_{\infty}$, we have $P_{L}(y)<A_{R}^{-1}(y)<P_{R}^{-1}(y)$, that is, $\Psi_{b}(y)>0$ for all $y>b$, so that system (4.1) has no periodic orbits.

(b3) Under the assumptions, we will apply the implicit function theorem at two different solutions of the equation

$$
\Psi_{b}(y)=b+P_{R}^{-1}(y)(y-b ; 0)-P_{L}(y)=0 .
$$

From Theorem 4.3(c) there is one unstable crossing periodic orbit for $b=0$. Therefore, there is a value $\bar{y}>0$ such that $(7.28)$ holds for $(b, y)=(0, \bar{y})$. From Lemma 7.6(a) we have $\Psi_{0}^{\prime}(\bar{y})>0$, and so, by the implicit function theorem, we can assure the existence of a smooth function $y=\delta(b)$, defined in a neighborhood of the origin, with $\delta(0)=\bar{y}$ such that $\Psi_{b}(\delta(b))=0$, so that there exists an unstable crossing periodic orbit for $b$ in such a neighborhood.

We know that $(7.28)$ also holds for $(y, b)=(0,0)$. If we try to apply the above reasoning at the point $(b, y)=(0,0)$, we find from $(7.27)$ that $\Psi_{0}^{\prime}(0)=0$, which is an obstacle to proceeding. However, from (7.25) we see that

$$
\frac{\partial}{\partial b} \Psi_{b}(y)=1-\left(P_{R}^{-1}\right)^{\prime}(y-b ; 0)
$$

Copyright $\odot$ by SIAM. Unauthorized reproduction of this article is prohibited. 
so that

$$
\left.\frac{\partial}{\partial b} \Psi_{b}(y)\right|_{(y, b)=(0,0)}=2
$$

Thus, considering analytical extensions of both functions $P_{R}^{-1}$ and $P_{L}$ if needed, we can assure the existence of a smooth function $b=\eta(y)$, defined in a neighborhood of 0 , with $\eta(0)=0$ and such that $\Psi_{\eta(y)}(y)=0$. Effectively, if $a_{R}<0<a_{L}$, then we have

$$
\eta^{\prime}(0)=-\left.\frac{\Psi_{b}^{\prime}(y)}{\frac{\partial \Psi_{b}}{\partial b}(y)}\right|_{(y, b)=(0,0)}=0
$$

since $\Psi_{0}^{\prime}(0)=0$, and computing the second derivative, after neglecting some vanishing terms, we get

$$
\eta^{\prime \prime}(0)=-\left.\frac{\Psi_{b}^{\prime \prime}(y)}{\frac{\partial \Psi_{b}}{\partial b}(y)}\right|_{(y, b)=(0,0)}=\frac{4}{3} \frac{a_{L} \gamma_{R}-a_{R} \gamma_{L}}{a_{R} a_{L}}>0 .
$$

If $a_{R} a_{L}=0$, we will assume $a_{R}<0$ and $a_{L}=0$ (otherwise apply symmetry $\Pi_{3}$ ), and we get $\eta^{\prime}(0)=1 / 2$. In the two cases $a_{L}>0$ and $a_{L}=0$ we have shown the existence of a crossing periodic orbit for $b=\eta(y)>0$ with $y$ positive and sufficiently small. Since for $a_{R}<0<a_{L}$ we have $\Psi_{0}(0)=0$ and $\Psi_{0}^{\prime \prime}(0)<0$, and as for $a_{R} a_{L}=0$ we have $\Psi_{0}^{\prime}(0)<0$, we must have $\Psi_{b}^{\prime}(y)<0$ for $b>0$ and sufficiently small. Hence the corresponding crossing periodic orbit is stable.

From Theorem 4.3(c) we also deduce that $\Psi_{0}(y)>0$ for all $y>\bar{y}$. Then by taking

$$
b>\max _{y \in[0, \bar{y}]}\left|\Psi_{0}(y)\right|
$$

and using Lemma 7.6(b), we can assure that for such values of $b$ there cannot exist a periodic orbit. The first assertion of the statement is proved.

If either $a_{R}=0$ or $a_{L}=0$, then from Proposition 7.3(f) or (g) we can assure that Poincaré map $P$ has a definite convexity. Hence, the Poincaré map has two fixed points at most. As for $b>0$ and small, there are two periodic orbits and none if $b$ is sufficiently big; by increasing $b$ we must pass through a value $b_{S N}$ satisfying the statement (b3).

A sketch of the proof for the case $a_{R}<0<a_{L}$ appears in Figure 7 .

(b4) When $\gamma_{R}+\gamma_{L}<0$, then $\lim _{y \rightarrow \infty} P^{\prime}(y)=e^{\left(\gamma_{R}+\gamma_{L}\right) \pi}<1$. Since $P(0)>b$, the Poincaré map has at least one fixed point $\bar{y}$ with $P^{\prime}(\bar{y})<1$, and so the system has a stable crossing periodic orbit.

In the following we will show the uniqueness of the crossing periodic orbit under additional assumptions. If $a_{R}=a_{L}=0$, then from (7.17) the Poincaré map has the unique fixed point

$$
\bar{y}=\frac{1+e^{\gamma_{R} \pi}}{1-e^{-\left(\gamma_{R}+\gamma_{L}\right) \pi}} b>b
$$

If $a_{R} a_{L}=0$ with $a_{R}+a_{L} \neq 0$, we can assume $a_{R}<0$ and $a_{L}=0$; otherwise apply symmetry $\Pi_{3}$. In this case, from Propositions 7.1 and 7.2 we get $\operatorname{sign} \Psi_{b}^{\prime \prime}(y)=\operatorname{sign}\left(P_{R}^{-1}\right)^{\prime \prime}(y)=\operatorname{sign} \gamma_{R}$, 

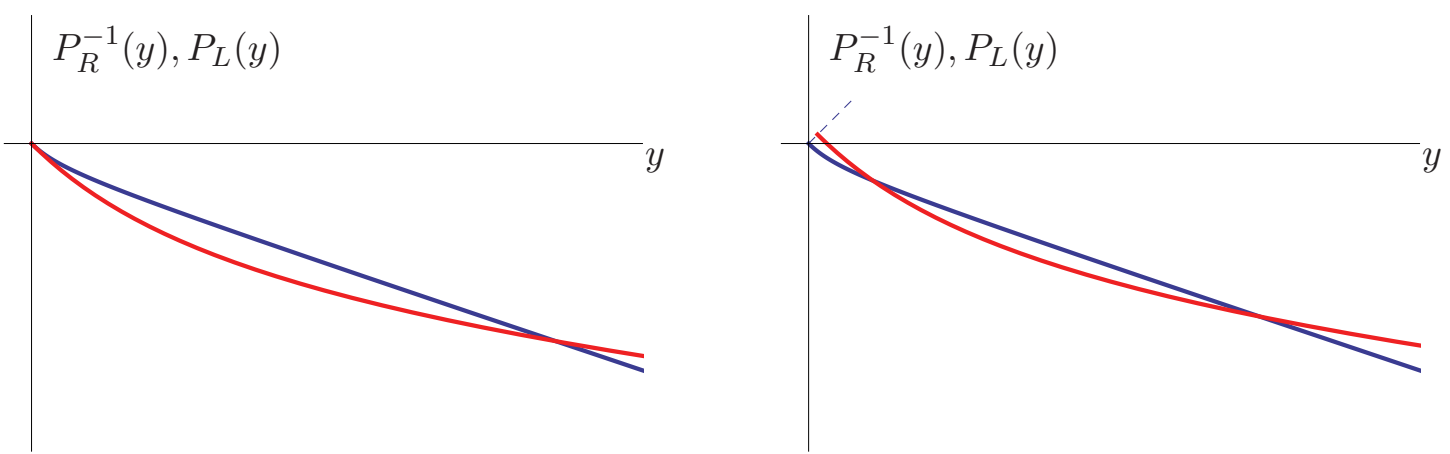

Figure 7. Sketch of the proof of Theorem 4.5(b3). In the left panel the situation for $b=0$ is represented, where map $P_{L}$ (blue line), with $\gamma_{L}<0$, cuts at map $P_{R}^{-1}$ (red line), with $\gamma_{R}>0$ at only one point outside the origin. In the right panel we have $b>0$, and now another intersection point appears.

so that the function $\Psi_{b}$ has a definite convexity. Two cases will be considered depending upon the sign of $\gamma_{R}$. If $\gamma_{R}<0$, then

$$
\lim _{y \rightarrow \infty} \Psi_{b}^{\prime}(y)=-e^{-\gamma_{R} \pi}+e^{\gamma_{L} \pi}=e^{-\gamma_{R} \pi}\left(-1+e^{\left(\gamma_{L}+\gamma_{R}\right) \pi}\right)<0,
$$

and taking into account $\Psi_{b}(b)>0$ and the convexity of function $\Psi_{b}$, the conclusion follows. If $\gamma_{R}>0$, then $\Psi_{b}^{\prime}(y)<-e^{-\gamma_{R} \pi}+e^{\gamma_{L} \pi}<0$, and the statement is shown.

Acknowledgment. The authors greatly acknowledge all the constructive suggestions of the associate editor and reviewers, which have improved the presentation and quality of the paper.

\section{REFERENCES}

[1] A. Andronov, A. Vitt, and S. Khaikin, Theory of Oscillations, Pergamon Press, Oxford, UK, 1966.

[2] V. Carmona, E. Freire, E. Ponce, and F. Torres, On simplifying and classifying piecewise-linear systems, IEEE Trans. Circuits Systems, 49 (2002), pp. 609-620.

[3] B. Coll, A. Gasull, and R. Prohens, Degenerated Hopf bifurcations in discontinuous planar systems, J. Math. Anal. Appl., 253 (2001), pp. 671-690.

[4] A. Colombo, P. Lamiani, L. Benadero, and M. Di Bernardo, Two-parameter bifurcation analysis of the buck converter, SIAM J. Appl. Dyn. Syst., 8 (2009), pp. 1507-1522.

[5] M. di Bernardo, C. J. Budd, A. R. Champneys, and P. Kowalczyk, Piecewise-Smooth Dynamical Systems: Theory and Applications, Appl. Math. Sci. 163, Springer-Verlag, London, 2008.

[6] M. Guardia, T. M. Seara, and M. A. Teixeira, Generic bifurcations of low codimension of planar Filippov systems, J. Differential Equations, 250 (2011), pp. 1967-2023.

[7] A. F. Filippov, Differential Equations with Discontinuous Right-Hand Sides, Kluwer Academic Publishers, Dordrecht, The Netherlands, 1988.

[8] E. Freire, E. Ponce, F. Rodrigo, And F. Torres, Bifurcation sets of continuous piecewise linear systems with two zones, Internat. J. Bifur. Chaos Appl. Sci. Engrg., 8 (1998), pp. 2073-2097.

[9] F. Giannakopoulos and K. Pliete, Closed trajectories in planar relay feedback systems, Dyn. Syst., 17 (2002), pp. 343-358.

[10] M. HAN AND W. Zhang, On Hopf bifurcation in non-smooth planar systems, J. Differential Equations, 248 (2010), pp. 2399-2416.

[11] S. HuAn AND X. YANG, On the number of limit cycles in general planar piecewise linear systems, Discrete Contin. Dynam. Systems, (2012), to appear.

Copyright (c) by SIAM. Unauthorized reproduction of this article is prohibited. 
[12] Y. Iwatani And S. Hara, Stability Analysis and Stabilization for Bimodad Piecewise Linear Systems Based on Eigenvalue Loci, Mathematical Engineering Technical Reports, 2004; available online at http://www.keisu.t.u-tokyo.ac.jp/research/techrep/data/2004/METR04-34.pdf.

[13] Yu. A. Kuznetsov, S. Rinaldi, and A. Gragnani, One-parameter bifurcations in planar Filippov systems, Internat. J. Bifur. Chaos Appl. Sci. Engrg., 13 (2003), pp. 2157-2188.

[14] J. Llibre And E. Ponce, Bifurcation of a periodic orbit from infinity in planar piecewise linear vector fields, Nonlinear Anal., 36 (1999), pp. 623-653.

[15] J. Llibre, E. Ponce, AND F. TORRes, On the existence and uniqueness of limit cycles in Liénard differential equations allowing discontinuities, Nonlinearity, 21 (2008), pp. 2121-2142.

[16] H. Sira-Ramírez and R. Silva-Ortigoza, Control Design Techniques in Power Electronic Devices, Springer-Verlag, London, 2006.

[17] J. J. StokeR, Nonlinear Vibrations in Mechanical and Electrical Systems, John Wiley, New York, 1950.

[18] S. Shui, X. Zhang, And J. Li, The qualitative analysis of a class of planar Filippov systems, Nonlinear Anal., 73 (2010), pp. 1277-1288.

[19] A. Tonnelier And W. Gerstner, Piecewise linear differential equations and integrate-and-fire neurons: Insights from two-dimensional membrane models, Phys. Rev. E, 67 (2003), 021908.

[20] Y. Zou, T. Kupper, And W. J. Beyn, Generalized Hopf bifurcations for planar Filippov systems continuous at the origin, J. Nonlinear Sci., 16 (2006), pp. 159-177.

Copyright $\odot$ by SIAM. Unauthorized reproduction of this article is prohibited. 